COMMUNICATIONS IN

ANALYSIS AND GEOMETRY

Volume 12, Number 1, 1-41, 2004

\title{
Dehn-Thurston Coordinates for Curves on Surfaces
}

\author{
Feng Luo And Richard Stong
}

\begin{abstract}
We prove that the geometric intersection numbers between two proper 1-dimensional submanifolds satisfy a Cauchy type inequality expressed in terms of the Dehn-Thurston coordinate. As an application, we reestablish the fundamental results in the theory of measured laminations on surfaces.
\end{abstract}

\section{Introduction.}

1.1. We study the space of isotopy classes of 1-dimensional submanifolds in a surface in this paper. The subject was originated by Max Dehn in his 1938 paper [De]. In this work, Dehn laid the foundation for the studies of the mapping class group of a surface and the space of isotopy classes of 1-dimensional submanifolds in a surface. According to Dehn, the most important proper 1-dimensional submanifolds are the curve systems which have the property that no component of the submanifold is null homotopic or homotopic into the boundary of the surface by homotopies relative to the boundary. Dehn defined the arithmetic field of a surface $\Sigma$, denoted by $C S(\Sigma)$, to be the set of all isotopy classes of curve systems. Dehn's main focus of study in [De] was the action of the mapping class group on the "arithmetic field" $C S(\Sigma)$. In 1976, William Thurston independently rediscovered the space $C S(\Sigma)$ and put one more vital ingredient into the study of "arithmetic field" $C S(\Sigma)$. Namely, the geometric intersection numbers between two isotopy classes of 1-dimensional submanifolds. Recall that if $\alpha$ and $\beta$ are isotopy classes of proper 1-dimensional submanifolds, then their geometric intersection number, denoted by $I(\alpha, \beta)$, is the minimal number of intersections between their representatives, i.e.,

$$
I(\alpha, \beta)=\min \{|a \cap b|: a \in \alpha, b \in \beta\} .
$$

Thurston developed the theory of measured laminations which is a topological completion of the pair $(C S(\Sigma), I)$ and used it to classify the elements in the mapping class group.

To study the arithmetic field $C S(\Sigma)$ of the surface, Dehn introduced a parameterization of it (the Dehn-Thurston coordinate, see $[\mathrm{PH}]$ or $\S 2$ for 
a definition). The parameterization is an injective homogeneous map $\Pi$ : $C S(\Sigma) \rightarrow\left(\mathbf{Z}^{2} / \pm\right)^{3 g+r-3} \times \mathbf{Z}_{\geq 0}^{r}$ where $g$ is the genus of the surface and $r \geq 0$ is the number of boundary component of the surface.

Our main result is a Cauchy inequality relating the geometric intersection number and the Dehn-Thurston coordinate. As an application, we shall reestablish some of the basic results in the theory of measured laminations from the inequality.

To state the main result, we need to introduce a natural metric on the space $\mathbf{Z}^{2} / \pm=\{[x, y] \mid x, y \in \mathbf{Z}$ so that $(x, y)$ and $-(x, y)$ are identified $\}$. We define the metric on the space $\mathbf{Z}^{2} / \pm$ to be $\left|\left[x_{1}, y_{1}\right]-\left[x_{2}, y_{2}\right]\right|=$ $\min \left(\left|x_{1}-x_{2}\right|+\left|y_{1}-y_{2}\right|,\left|x_{1}+x_{2}\right|+\left|y_{1}+y_{2}\right|\right)$ and $|[x, y]|=|[x, y]-[0,0]|$. The metric on the product space $\left(\mathbf{Z}^{2} / \pm\right)^{k} \times \mathbf{Z}^{r}$ is defined to be the product metric $\left|\left(v_{1}, \ldots, v_{k+r}\right)-\left(u_{1}, \ldots, u_{k+r}\right)\right|=\sum_{i=1}^{k+r}\left|v_{i}-u_{i}\right|$. Also $\left|\left(v_{1}, \ldots, v_{k+r}\right)\right|=$ $\sum_{i=1}^{k+r}\left|v_{i}\right|$.

Theorem. For a compact oriented surface $\Sigma$, the geometric intersection number satisfies the Cauchy inequality that

$$
|I(\alpha, \beta)-I(\alpha, \gamma)| \leq 3|\Pi(\alpha)||\Pi(\beta)-\Pi(\gamma)|
$$

for all $\alpha, \beta, \gamma \in C S(\Sigma)$. Here $\Pi(\alpha)$ is the Dehn-Thurston coordinate of $\alpha$.

If the 3-holed sphere decomposition used in the definition of the DehnThurston coordinate contains only embedded 4-holed spheres, then

$$
|I(\alpha, \beta)-I(\alpha, \gamma)| \leq 2|\Pi(\alpha)||\Pi(\beta)-\Pi(\gamma)|
$$

Furthermore, the inequality (1.2) is sharp.

Here by a 3 -holed sphere decomposition containing only 4-holed spheres, we mean any two 3-holed spheres in the decomposition do not share more than one boundary components (see figure 5.1 (b), (c)). For these 3-holed sphere decompositions, the inequality (1.2) is sharp. Indeed, for the 4-holed sphere $\Sigma_{0,4}$, if one takes $\alpha, \beta$, and $\gamma$ as shown in figure 1.1, then the inequality (1.2) becomes an equality. It is possible that inequality (1.1) is also sharp. However, we have not been able to find an example realizing the equality in (1.1).

We remark that in [Re], using the theory of train-tracks, it is proved that the inequality $|I(\alpha, \beta)-I(\alpha, \gamma)| \leq K|\Pi(\alpha)||\Pi(\beta)-\Pi(\gamma)|$ holds for three classes $\alpha, \beta$ and $\gamma$ carried by a train-track and the constant $K$ depends on the train track. 

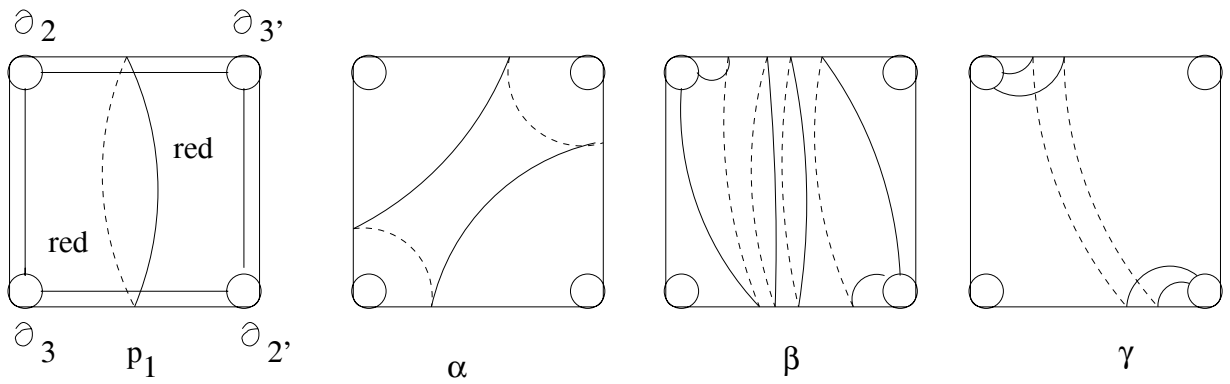

Figure 1.1

1.2. As an application, we shall reestablish some of the basic results in the theory of measured laminations which is a topological completion of the pair $(C S(\Sigma), I)$. Thurston first observed that the pairing $I: C S(\Sigma) \times C S(\Sigma) \rightarrow \mathbf{Z}$ is similar to a non-degenerate bilinear form on a lattice in the sense that (1) for any $\alpha$ in $C S(\Sigma)$ there is $\beta$ in $C S(\Sigma)$ so that $I(\alpha, \beta) \neq 0$, and (2) $I\left(\alpha^{j}, \beta^{k}\right)=j k I(\alpha, \beta)$ where $\alpha^{j}$ is the collection of $j$ copies of $\alpha$. In linear algebra, given a non-degenerate bilinear form on a lattice $L$ of rank $r$, there is a canonical completion of the lattice to a Euclidean space so that the bilinear form extends naturally. The theory of measured laminations is the exact analogy. Define an embedding $\Theta$ of $C S(\Sigma)$ to $\mathbf{R}^{C S(\Sigma)}$ by sending $\alpha$ to the function $\Theta(\alpha)=I(\alpha,$.$) , i.e., \Theta(\alpha)(\beta)=I(\alpha, \beta)$. Then the space of measured laminations $M L(\Sigma)$ is defined to be the closure of $\mathbf{Q}_{\geq 0} \times \Theta(C S(\Sigma))$ in the product topology. The basic theorem in the theory of measured laminations is the following remarkable result which shows in particular that the geometric intersection numbers behave very well in dimension 2 .

Theorem. ([Th1],[Th2], [Bo]) For each compact orientable surface $\Sigma$ of genus $g$ with $r$ many boundary components,

(1) the intersection pairing I extends to a continuous homogeneous map from $M L(\Sigma) \times M L(\Sigma)$ to $\mathbf{R}$, and $\mathbf{R}_{\geq 0}^{r}$.

(2) the space $M L(\Sigma)$ is homeomorphic to the Euclidean space $\mathbf{R}^{6 g+r-3} \times$

See $[\mathrm{Bo}],[\mathrm{FLP}],[\mathrm{HM}],[\mathrm{PH}]$ and others for proofs. It will be shown in $\S 6$ that this result follows from theorem 1.1 and a fact that the twisting coordinate can be expressed as a universal function in terms of the geometric intersection numbers with a fixed set of simple loops (Prop. 4.4).

1.3. The paper is organized as follows. In $\S 2$, we recall the definition of Dehn-Thurston coordinate. We shall make an extensive use of a natural multiplicative structure on the space $C S(\Sigma)$ to define the twisting coordinate. 
In $\S 3$, we study the Dehn-Thurston coordinate and the multiplication of curve systems on the 3 -holed sphere. The corresponding results for the 1holed torus and the 4-holed sphere are proved in section $\S 4$. In $\S 5$, we prove theorem 1.1 and in $\S 6$, we derive the basic result on the space of measured laminations from theorem 1.1.

1.4. Acknowledgment. The work is supported in part by the NSF.

\section{Dehn-Thurston Coordinate of Curve Systems.}

We will define the Dehn-Thurston coordinate on $C S(\Sigma)$ in this section. The Dehn-Thurston coordinate on surfaces with non-negative Euler number is very simple and will not be discussed here. Thus unless mentioned otherwise, we will always assume that the surface $\Sigma$ is oriented with negative Euler number in this section. The basic ingredient to define the coordinate is a decomposition of the surface into hexagons (an H-decomposition). This will be defined in section 2.2 .

This section is organized as follows. In $\S 2.1$, we introduce the notations and conventions to be used in the paper. In $\S 2.2$, we recall the classification of the curve systems in the 3 -holed sphere and introduce the concept of standard curve systems. In $§ 2.3$, we define the P-decomposition (pantsdecomposition) and H-decomposition (hexagonal-decomposition) of a surface. In $\S 2.4$, we recall a very useful multiplicative structure on the space $C S(\Sigma)$ and list its basic properties. The Dehn-Thurston coordinate is defined in $\S 2.5$. There are some overlaps between the work of $[\mathrm{PH}]$ and ours in the approach to the Dehn-Thurston coordinate. The use of hexagonaldecomposition in this paper seems to be graphically easier to understand than that of $[\mathrm{PH}]$.

2.1. We shall use the following notation and conventions. Let $\Sigma_{g, r}$ be the orientable compact surface of genus $g$ with $r \geq 0$ boundary components. The interior of a surface $X$ will be denoted by $\operatorname{int}(X)$. If a surface $X$ is oriented, then all its subsurfaces have the induced orientation. We will always draw oriented surface so that its orientation is the right-hand orientation in the front face. If $s$ is a proper submanifold of a surface, we use $N(s)$ to denote a small tubular neighborhood of $s$. The isotopy class of a submanifold $s$ is denoted by $[s]$. If $a, b$ are curve systems, we will also use $I(a, b)$ or $I([a], b)$ or $I(a,[b])$ to denote the intersection number $I([a],[b])$. Two isotopy classes $\alpha$ and $\beta$ in $C S(\Sigma)$ are called disjoint if $I(\alpha, \beta)=0$. If $I(\alpha, \beta) \neq 0$, we say $\alpha$ intersects $\beta$. If $s$ is a component of a curve system $a$, we will call the isotopy class $[s]$ a component of $[a]$. A parallel copy of a curve system $a$ is a new curve system $a^{\prime}$ isotopic to $a$ so that $a^{\prime}$ and $a$ are disjoint and $a^{\prime} \subset N(a)$. When 
a curve system $a$ is written as a union $a_{1} \cup \ldots \cup a_{n}$, it is always understood that each $a_{i}$ is a union of components of $a$. Let $2 \mathbf{Z}$ be the set of all even numbers. The isotopic submanifolds $a, b$ will be denoted by $a \cong b$.

A function $f: \mathbf{R}^{n} \rightarrow X$ where $X$ admits an action of $\mathbf{R}_{>0}$ is called homogeneous if for all $k \in \mathbf{R}_{>0}, f(k x)=k f(x)$.

2.2. We begin with the classification of the curve systems on the 3-holed sphere $\Sigma_{0,3}$. It is well known that the curve systems in $\Sigma_{0,3}$ are classified up to isotopy by their intersection numbers with the three boundary components. See for instance [FLP]. To be more precise, let the boundary components of the 3 -holed sphere $\Sigma_{0,3}$ be $\partial_{1}, \partial_{2}, \partial_{3}$. Define a map $\Pi: C S\left(\Sigma_{0,3}\right) \rightarrow\left\{\left(x_{1}, x_{2}, x_{3}\right) \in \mathbf{Z}_{\geq 0}^{3} \mid x_{1}+x_{2}+x_{3} \in 2 \mathbf{Z}\right\}$ by sending $\alpha$ to $\left(I\left(\alpha, \partial_{1}\right), I\left(\alpha, \partial_{2}\right), I\left(\alpha, \partial_{3}\right)\right)$. Then the map $\Pi$ is a bijection. This is the Dehn-Thurston coordinate for the 3 -holed sphere. One can list the curve system with coordinate $\left(x_{1}, x_{2}, x_{3}\right)$ as in figure $2.1(\mathrm{a})$.

An $H$-decomposition on the surface $\Sigma_{0,3}$ is a curve system $b_{1} \cup b_{2} \cup b_{3}$ whose Dehn-Thurston coordinate is $(2,2,2)$. We call the closure of each component of $\Sigma_{0,3}-b_{1} \cup b_{2} \cup b_{3}$ a hexagon whose boundary consists of three edges in $b_{1} \cup b_{2} \cup b_{3}$ and the other three in $\partial \Sigma_{0,3}$. We assume that $b_{i}$ is disjoint from $\partial_{i}$.

Fix an orientation on the surface $\Sigma_{0,3}$ and color one of the hexagons red. Then with respect to this colored H-decomposition of the oriented surface $\Sigma_{0,3}$, each element $\alpha \in C S\left(\Sigma_{0,3}\right)$ has a standard representing curve system. Here a curve system is called standard if each component of it is standard. An arc $a$ is standard if either it lies entirely in the red-hexagon or if $\partial a \subset \partial_{i}$, then $\partial a$ is in the red-hexagon and $\left|a \cap\left(b_{1} \cup b_{2} \cup b_{3}\right)\right|=2=\left|a \cap\left(b_{i} \cup b_{j}\right)\right|$ so that the cyclic order of the sets $\left(a \cap \partial_{i}, a \cap b_{i}, a \cap b_{j}\right)$ in the boundary of the redhexagon coincides with the induced orientation from the red-hexagon. For instance the standard representatives of the curve system with coordinate $\left(x_{1}, x_{2}, x_{3}\right)$ are listed in figure $2.1(\mathrm{~b})$.

2.3. Let $C S_{0}(\Sigma) \subset C S(\Sigma)$ be the subset consisting of isotopy classes of curve systems which contain no arc components. We call an element $[p] \in C S_{0}(\Sigma)$ a $P$-decomposition if each component of the surface $\Sigma-\cup_{i=1}^{k} \operatorname{int}(N(p))$ is a 3 -holed sphere. In this case, the curve system $p$ must have exactly $3 g+r-3$ many components. An $H$-decomposition of the surface is a pair $\left.([p],[b]) \in C S_{0}(\Sigma) \times C S(\Sigma)\right)$ so that (1) $[p]$ is a $\mathrm{P}$-decomposition, (2) $I(p,[b])=|p \cap b|$, and (3) for each 3 -holed sphere $P$ bounded by components of $p,(P, P \cap b)$ is an $\mathrm{H}$-decomposition of the 3 -holed sphere. 


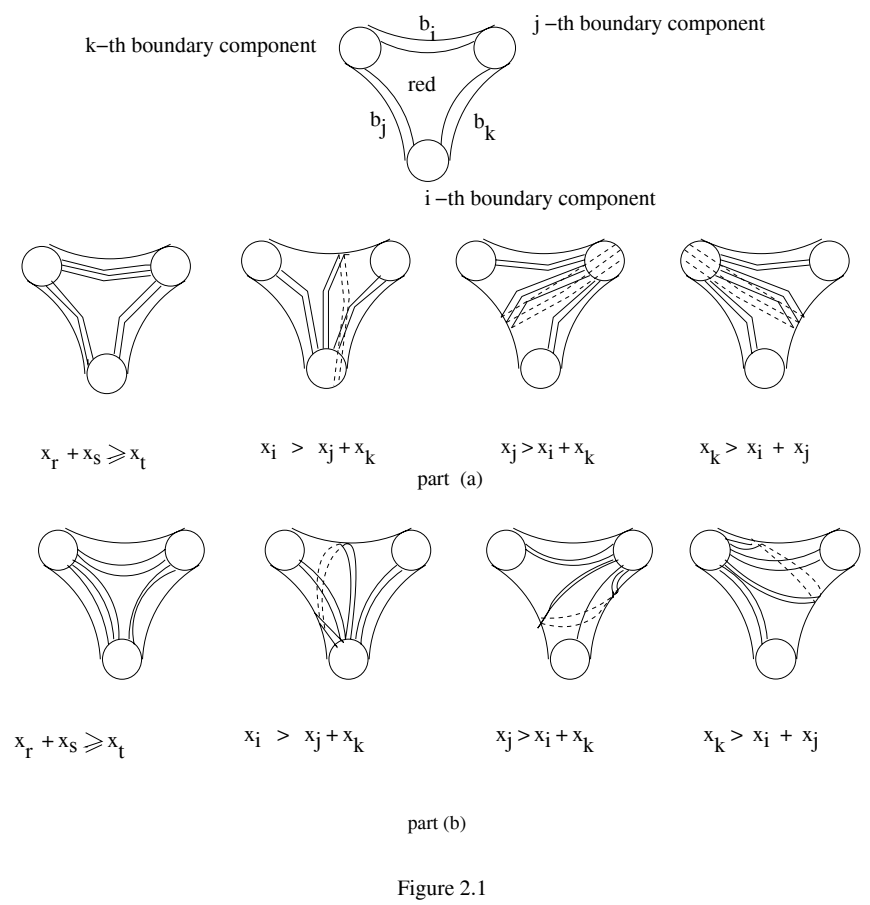

In this paper, we shall define Dehn-Thurston coordinate associated to colored H-decompositions which satisfy the condition that one can color the set of all hexagons in the decomposition into red and white so that there is exactly one red-hexagon in each 3-holed sphere and that red and white hexagons never share the same $p$-edges (recall that each hexagon has six edges either in $p \cup \partial \Sigma$ or in $b$.)

2.4. Let us recall the concept of multiplication of two curve systems in $C S(\Sigma)$. See [Bo1], $[\mathrm{Lu}]$, and $[\mathrm{Pa}]$. Given $\alpha$ and $\beta$ in $C S(\Sigma)$, take $a \in \alpha$ and $b \in \beta$ so that $|a \cap b|=I(\alpha, \beta)$. If $\alpha$ and $\beta$ are disjoint, we define $\alpha \beta$ to be $[a \cup b]$. If $I(\alpha, \beta)>0$, then $\alpha \beta$ is define to be the isotopy class of the 1-dimensional submanifold $a b$ obtained by resolving all intersection points in $a \cap b$ from $a$ to $b$. Here by the resolution from $a$ to $b$ we mean the following surgery. At each point $p \in a \cup b$, fix any orientation on $a$. Then use the orientation of the surface to determine an orientation of $b$ at $p$ at $p$. Finally resolve the singularity at $p$ according to the orientations on $a$ and $b$. One checks easily that this is independent of the choice of orientation on $a$. See figure 2.2. 


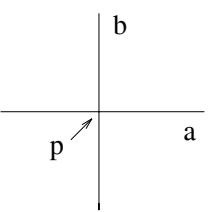

The right-hand orientation on the front face

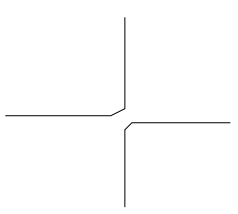

$\mathrm{ab}$

Figure 2.2

It is shown in $[\mathrm{Lu}]$ that the 1-dimensional submanifold $a b$ is still a curve system. Also if $\alpha$ is the isotopy class of a simple loop, then $\alpha^{I(\alpha, \beta)} \beta$ is the positive Dehn twist along $\alpha$ applied to $\beta$.

Here is an example illustrating the use of the multiplication.

Example. If $a$ and $b$ are curve systems in the annulus $\Sigma_{0,2}=S^{1} \times[-1,1]$ with central curve $c=S^{1} \times 0$ so that $\partial a=\partial b$, then there exists a nonnegative integer $k$ so that either $a \cong c^{k} b$ or $a \cong b c^{k}$ by an isotopy leaving the boundary $\partial \Sigma_{0,2}$ fixed.
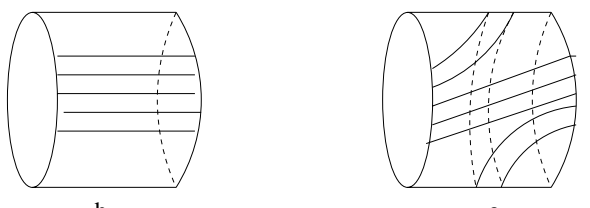

a

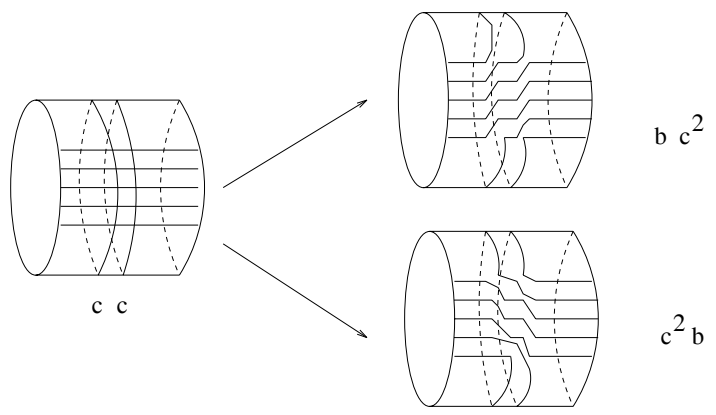

two resolutions

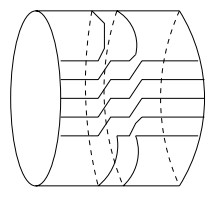

b $c^{2}$

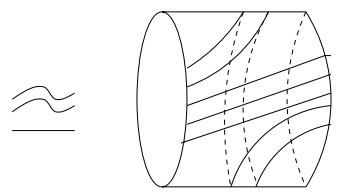

a

Figure 2.3 
The basic properties of the multiplication are obtained in $[\mathrm{Lu}]$ and can be summarized in the following theorem. Since the paper $[\mathrm{Lu}]$ has not appeared in print, we present a proof of it in the appendix for completeness.

Theorem. The multiplication $C S(\Sigma) \times C S(\Sigma) \rightarrow C S(\Sigma)$ sends $C S_{0}(\Sigma) \times$ $C S_{0}(\Sigma) \rightarrow C S_{0}(\Sigma)$ and has the following properties.

(1) It is preserved under the action of the orientation preserving homeomorphisms of the surface.

(2) (commutativity) If $I(\alpha, \beta)=0$, then $\alpha \beta=\beta \alpha$. If $\alpha \beta=\beta \alpha$ and $\alpha \in C S_{0}(\Sigma)$, then $I(\alpha, \beta)=0$.

(3) (associativity) Suppose $\alpha, \beta$, and $\gamma$ are in $C S(\Sigma)$ so that the ordered set $(\alpha, \beta, \gamma)$ contains no positive triangles and positive quadrilaterals, then $\alpha(\beta \gamma)=(\alpha \beta) \gamma$.

(4) (inverse) If $\alpha$ is in $C S_{0}(\Sigma)$ and each component of $\alpha$ intersects $\beta$, then $\alpha(\beta \alpha)=(\alpha \beta) \alpha=\beta$. Furthermore, $I(\alpha, \beta)=I(\alpha, \alpha \beta)=I(\alpha, \beta \alpha)$.

(5) For any integer $k \in \mathbf{Z}_{>0},(\alpha \beta)^{k}=\alpha^{k} \beta^{k}$.

(6) (triangle inequality) $I(\alpha, \beta \gamma) \leq I(\alpha, \beta)+I(\alpha, \gamma)$. The inequality becomes an equality if $(\alpha, \beta, \gamma)$ contains no positive triangles or quadrilaterals. Furthermore, if $\beta \in C S_{0}(\Sigma)$, then $|I(\alpha, \beta \gamma)-I(\alpha, \gamma)| \leq I(\alpha, \beta)$ and $|I(\alpha, \gamma \beta)-I(\alpha, \gamma)| \leq I(\alpha, \beta)$.

Here we say three ordered elements $\alpha_{1}, \alpha_{2}, \alpha_{3} \in C S(\Sigma)$ contain a positive triangle or a positive quadrilateral if the following condition is satisfied. Take $a_{i} \in \alpha_{i}$ so that $\left|a_{i} \cap a_{j}\right|=I\left(a_{i}, a_{j}\right)$ and $a_{1} \cap a_{2} \cap a_{3}=\emptyset$. There is a topological disc $D$ which is the closure of a component of $\Sigma-\cup_{i=1}^{3} a_{i}$ so that the boundary $\partial D$ is a union of three (resp. four) $\operatorname{arcs} c_{1}, c_{2}, c_{3}$ (resp. $\left.c_{1}, c_{2}, c_{3}, d\right)$ with $c_{i} \subset a_{i}, d \subset \partial \Sigma$ and the cyclic order $\left(c_{1}, c_{2}, c_{3}\right)$ coincides with the induced orientation on $\partial D$ from $D$. See figure 2.4

2.5. Fix a colored H-decomposition $\left(\left[p_{1} \cup \ldots \cup p_{3 g+r-3}\right],[b]\right)$ of an oriented surface $\Sigma=\Sigma_{g, r}$. The Dehn-Thurston coordinate of $\alpha$ in $C S(\Sigma)$ is a vector in $\left(\mathbf{Z}^{2} / \pm\right)^{3 g+r-3} \times \mathbf{Z}_{\geq 0}^{r}$. Let us write the image of a vector $\left(v_{1}, \ldots, v_{k}\right) \in\left(\mathbf{Z}^{2}\right)^{k}$ in $\left(\mathbf{Z}^{2} / \pm\right)^{k}$ by $\left(\left[v_{1}\right], \ldots,\left[v_{k}\right]\right)$. Then

$$
\Pi(\alpha)=\left(\left[x_{1}, t_{1}\right], \ldots,\left[x_{3 g+r-3}, t_{3 g+r-3}\right], x_{3 g+r-2}, \ldots, x_{3 g+2 r-3}\right) .
$$

For simplicity, let us use $p_{3 g+r-3+j}=\partial_{j} \Sigma$. Then the $i$-th intersection coordinate $x_{i}$ is defined to be $I\left(\alpha, p_{i}\right)$ for $1 \leq i \leq 3 g+2 r-3$. 

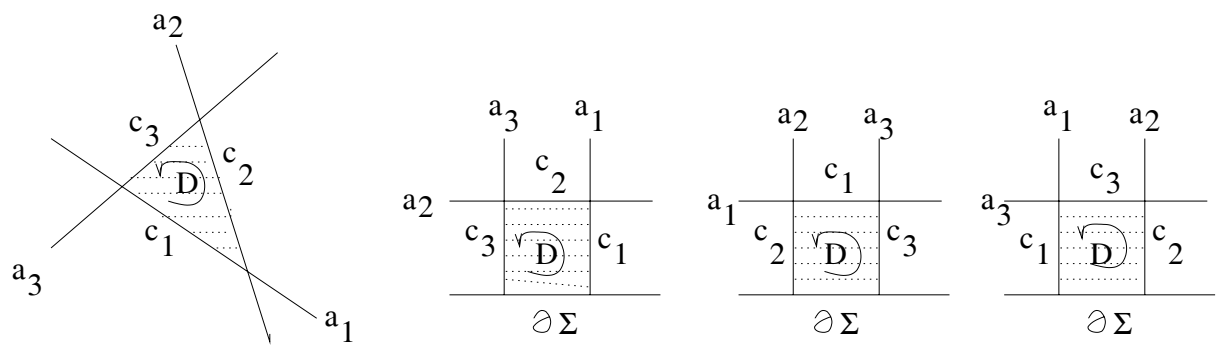

Positive triangles and postive quadrilaterals

right-hand orientation on the front face

Figure 2.4

The definition of the $i$-th twisting coordinate $t_{i}$ for $1 \leq i \leq 3 g+r-3$ is more complicated. By definition, if $p_{i}, p_{j}$ and $p_{k}$ (two of them may be the same) bound a 3 -holed sphere $P$ in $\Sigma$, then $x_{i}+x_{j}+x_{k}$ is an even number. Thus by fixing $x_{i}$ points in the red-arc part of $p_{i}$, we can construct a standard curve system on the 3 -holed sphere with coordinate $\left(x_{i}, x_{j}, x_{k}\right)$ having these fixed $x_{m}$ points in $p_{m},(m=i, j, k)$ as end points. The union of all these standard curve systems produces a curve system $s$ in $\Sigma$ so that $I\left(s, p_{i}\right)=x_{i}$. We call the curve system $s$ standard and its isotopy class $[s]$ the zero-twisting representative of $\alpha$ and denote it by $\alpha_{z t}$.

For simplicity, we shall use $\alpha^{n} \beta$ to denote $\beta \alpha^{-n}$ if $n$ is a negative integer. For instance, example 2.4 concludes that $a \cong c^{k} b$ for some $k \in \mathbf{Z}$. By the associativity and the inverse property of theorem 2.3, we have that $\alpha^{m}\left(\alpha^{n} \beta\right)=\alpha^{m+n} \beta$ for all $m, n \in \mathbf{Z}$ if each components of $\alpha$ intersects $\beta$.

Lemma. Each $\alpha$ in $C S(\Sigma)$ can be expressed uniquely as

$$
\alpha=\left[p_{1}^{t_{1}} \ldots p_{3 g+r-3}^{t_{3 g+r-3}}\right] \alpha_{z t}
$$

where $t_{i} \in \mathbf{Z}$ so that if $I\left(\alpha, p_{i}\right)=0$ then $t_{i} \geq 0$.

Proof. By the classification of curve systems in the 3-holed sphere, we may choose small regular neighborhood $N=N\left(p_{1} \cup \ldots \cup p_{3 g+r-3}\right)=\cup_{i=1}^{3 g+r-3} N\left(p_{i}\right)$ and a representative $a$ of $\alpha$ so that (1) $a$ and $s$ coincide outside the neighborhood $N$ and (2) $a \cap N$ and $s \cap N$ are curve systems in $N$ having the same end points. Now for each index $i$, consider the curve systems $a \cap N\left(p_{i}\right)$ and $s \cap N\left(p_{i}\right)$. By example 2.4, we find an integer $t_{i}$ so that $a \cap N\left(p_{i}\right)$ is isotopic to $p_{i}^{t_{i}}\left(s \cap N\left(p_{i}\right)\right)$ by an isotopy which is the identity map on $\partial N\left(p_{i}\right)$. 
Furthermore, if $I\left(\alpha, p_{i}\right)=0$ then $t_{i}$ is the number of components of $\alpha$ which are $\left[p_{i}\right]$. Thus we see that equation (2.1) holds.

To show that the numbers $t_{i}$ are unique, we use the triangle inequality in theorem 2.4. Suppose otherwise, then there exists $\alpha$ in $C S(\Sigma)$ and a non-zero vector $\left(t_{1}, \ldots t_{3 g+r-3}\right) \in \mathbf{Z}^{3 g+r-3}$ so that

$$
\Pi_{i=1}^{3 g+r-3}\left[p_{i}^{t_{i}}\right] \alpha=\alpha .
$$

Let us separate the exponents $t_{i}$ into positive and negative parts. Say $I_{+}=$ $\left\{i \mid t_{i}>0\right\}$ and $I_{-}=\left\{i \mid t_{i}<0\right\}$. Then we have

$$
\left(\Pi_{i \in I_{+}}\left[p_{i}^{t_{i}}\right]\right) \alpha\left(\Pi_{i \in I_{-}}\left[p_{i}^{-t_{i}}\right]\right)=\alpha .
$$

Applying the above equation $n$ times by multiplying $\Pi_{i \in I_{+}}\left[p_{i}^{t_{i}}\right]$ from the left and $\Pi_{i \in I_{-}}\left[p_{i}^{-t_{i}}\right]$ from the right and use the associativity (theorem 2.4 (3)), we obtain

$$
\left(\Pi_{i \in I_{+}}\left[p_{i}^{n t_{i}}\right]\right) \alpha\left(\Pi_{i \in I_{-}}\left[p_{i}^{-n t_{i}}\right]\right)=\alpha .
$$

Without loss of generality, we assume that $t_{1}>0$. Choose a simple loop $\beta \in C S_{0}(\Sigma)$ so that $I\left(\beta, p_{1}\right)>0$ and $\beta$ is disjoint from all other $p_{i}$ 's. By theorem $2.4(6)$ that $I(x, y z) \geq \max (I(x, y)-I(x, z), I(x, z)-I(x, y))$ for $y \in C S_{0}(\Sigma)$, we have,

$$
\begin{aligned}
I(\beta, \alpha) & =I\left(\beta,\left(\Pi_{i \in I_{+}}\left[p_{i}^{n t_{i}}\right]\right) \alpha\left(\Pi_{i \in I_{-}}\left[p_{i}^{-n t_{i}}\right]\right)\right) \\
& \geq I\left(\beta,\left(\Pi_{i \in I_{+}}\left[p_{i}^{n t_{i}}\right]\right) \alpha\right)-I\left(\beta, \Pi_{i \in I_{-}}\left[p_{i}^{-n t_{i}}\right]\right) \\
& =I\left(\beta,\left(\Pi_{i \in I_{+}}\left[p_{i}^{n t_{i}}\right]\right) \alpha\right) \\
& \geq I\left(\beta, \Pi_{i \in I_{+}}\left[p_{i}^{n t_{i}}\right]\right)-I(\beta, \alpha) \\
& =n t_{1} I\left(\beta,\left[p_{1}\right]\right)-I(\beta, \alpha) .
\end{aligned}
$$

Since this has to be true for all $n$, we obtained a contradiction. QED

We call $t_{i}$ the $i$-th twisting coordinate of the Dehn-Thurston coordinate of $\alpha$. The $i$-th Dehn-Thurston coordinate of $\alpha$ is $\left[x_{i}, t_{i}\right]$ for $i \leq 3 g+r-3$ and is $x_{i}$ for $i>3 g+r-3$. By definition, the space $C S_{0}(\Sigma)$ has zero $i$-th Dehn-Thurston coordinates for $i \geq 3 g+r-2$.

Let us summarize what we have proved so far in the following proposition.

Proposition. The Dehn-Thurston coordinate is a homogeneous bijection $\Pi: C S(\Sigma) \rightarrow\left\{\left(\left[x_{1}, t_{1}\right], \ldots,\left[x_{3 g+r-3}, t_{3 g+r-3}\right], x_{3 g+r-2}, \ldots, x_{3 g+2 r-3}\right) \in\right.$ $\left(\mathbf{Z}^{2} / \pm\right)^{2 g+r-3} \times\left(\mathbf{Z}_{\geq 0}\right)^{r} \mid$ if $p_{i}, p_{j}$ and $p_{k}$ bound a 3-holed sphere, then $x_{i}+$ $\left.x_{j}+x_{k} \in 2 \mathbf{Z}\right\}$. 
Proof. We have established all assertions except the homogeneity. Suppose $\alpha$ is in $C S(\Sigma)$. For all $k \in \mathbf{Z}_{\geq 0}, I\left(\alpha^{k}, p_{i}\right)=k I\left(\alpha, p_{i}\right)$. This shows that $\left(\alpha^{k}\right)_{z t}=\left(\alpha_{z t}\right)^{k}$ by definition. Combining this with theorem 2.4, we see that $\Pi\left(\alpha^{k}\right)=k \Pi(\alpha)$.

2.6. As an example, let us calculate the Dehn-Thurston coordinates of $\alpha, \beta$ and $\gamma$ on the 4-holed sphere with an $\mathrm{H}$-decomposition given by figure 1.1. Then $\Pi(\alpha)=([2,0], 0,0,0,0), \Pi(\beta)=([0,-2], 2,0,0,2)$ and $\Pi(\gamma)=([2,-2], 2,0,0,2)$. Thus $|\Pi(\alpha)|=2$ and $|\Pi(\beta)-\Pi(\gamma)|=2$. But we also have $I(\alpha, \beta)=8, I(\alpha, \gamma)=0$. This shows that the inequality in theorem 1.1 is sharp.

2.7. For applications, we have to consider a slightly refined version of curve systems and isotopies. Suppose $\Sigma$ is a surface so that each boundary component of it contains a red interval. We say a curve system in $\Sigma$ good if its boundary components are in the red intervals. The space of all isotopy classes of good curve systems where isotopies leave red interval invariant is denoted by $C S^{g}(\Sigma)$.

Note that there is a natural forgetful map $F: C S^{g}(\Sigma) \rightarrow C S(\Sigma)$ which is a surjection so that $F(x)=F(y)$ if and only if these two good curve systems differ by some twists along the boundary components $\partial_{i}$ of the surface, i.e., $x \cong \Pi_{i=1}^{r} \partial_{i}^{t_{i}} y$ by an isotopy leaving all red-arcs in the boundary invariant. Here by $\partial_{i}^{n} y$ we mean $\left(\partial_{i}^{\prime}\right)^{n} y$ where $\partial_{i}^{\prime}$ is a parallel copy of $\partial_{i}$. See figure 2.5 .

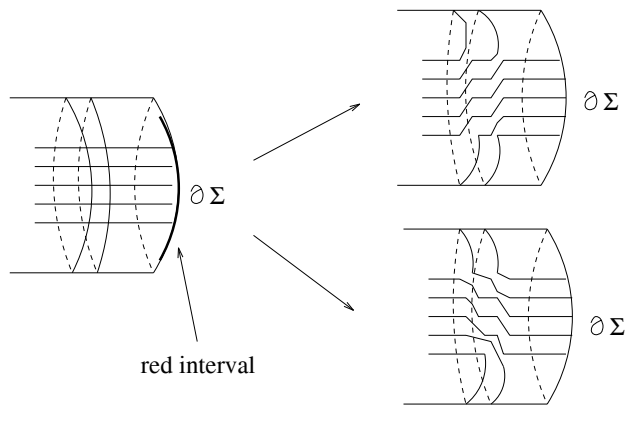

Twisting at the boundary components

Figure 2.5

Note that the Dehn-Thurston coordinate of $C S^{g}(\Sigma)$ can be defined in the same way and is a homogeneous injection $\Pi: C S^{g}(\Sigma) \rightarrow\left(\mathbf{Z}^{2} / \pm\right)^{3 g+r-3} \times$ $\left(\mathbf{Z}_{\geq 0} \times \mathbf{Z}\right)^{r}$. To be more precise, let us fix a colored H-decomposition of the surface so that the red-intervals in the boundary are exactly the intersection of the boundary with the red-hexagons. Then each good curve system in 
the surface is isotopic to a good curve system of the form

$$
\left[p_{1}^{t_{1}} \ldots p_{3 g+2 r-3}^{t_{3 g+2 r-3}}\right] \alpha_{z t}
$$

where $p_{3 g+r-3+j}=\partial_{j}$ is the $j$-th boundary component and $\alpha_{z t}$ is the zerotwisting class. The only novelty in this case is that we can define the twisting coordinate of a good curve system with respect to each boundary component of the surface.

2.8. Main idea of the proof. We sketch the proof of the main theorem 1.1 in this subsection. For simplicity, we will focus on the proof of inequality (1.1). First of all, by homogeneity that $I\left(\alpha, \beta^{2}\right)=2 I(\alpha, \beta)$ and $\pi\left(\beta^{2}\right)=$ $2 \pi(\beta)$, it suffices to prove (1.1) for classes $\beta$ and $\gamma$ so that $\pi(\beta)=u$ and $\pi(\gamma)=v$ are even vectors, i.e., all $x_{i}$ and $t_{i}$ coordinates of them are even integers. Now given any two even vectors $u$ and $v$ so that their distance $|u-v|=2 n$, there exists a sequence of $n+1$ even vectors $u_{0}=u, u_{1}, \ldots, u_{n}=v$ so that $\left|u_{i}-u_{i+1}\right|=2$. On the other hand, by proposition 2.5 , each even vector $u_{i}$ is the image $\pi\left(\beta_{i}\right)$ for some $\beta_{i} \in C S(\Sigma)$. Thus by elementary interpolation, it suffices to prove inequality (1.1) for classes $\beta$ and $\gamma$ so that $\pi(\beta)$ and $\pi(\gamma)$ are even vectors of distance two apart. This means that the Dehn-Thurston coordinates of $\beta$ and $\gamma$ are the same except at one $x_{i}$ - or $t_{j-}$ coordinate which differs by 2 . In this case, we prove that $\beta$ and $\gamma$ are related by a multiplication by at most five simple loops $\beta=\delta_{1} \ldots \delta_{s} \gamma \delta_{s+1} \ldots \delta_{t}$ where $t \leq 5$ so that $\delta_{i}$ 's are quite simple. In fact, these simple loops $\delta_{i}$ 's satisfy the inequality that for all $\alpha \in C S(\Sigma)$,

$$
\sum_{i=1}^{t} I\left(\alpha, \delta_{i}\right) \leq 6|\Pi(\alpha)|
$$

Thus by the triangle inequality (theorem 2.4(6)),

$$
|I(\alpha, \beta)-I(\alpha, \gamma)| \leq \sum_{i=1}^{t} I\left(\alpha, \delta_{i}\right) \leq 6|\Pi(\alpha)|=3|\Pi(\alpha)||\Pi(\beta)-\Pi(\gamma)|
$$

More precisely if $\beta$ and $\gamma$ differ by 2 in a $t_{j}$-th twisting coordinate and all other coordinates are the same, then by definition we have $\beta=\left[p_{j}\right]^{t} \delta$ where $t=2$ or -2 and $I\left(\alpha, p_{j}^{|t|}\right)=2 x_{j}(\alpha) \leq 2|\Pi(\alpha)|$. Thus inequality (1.1) follows easily in this case. The difficult case is the change of some $x_{i}$-th intersection coordinate by 2 . This will be the main focus of study in sections 3 and 4 . 


\section{Computations on the 3-holed Sphere.}

3.1. In this section, we will carry out the computation of the intersection number and the Dehn-Thurston coordinate of the multiplications of curve systems on the 3 -holed sphere. The goal is to prove proposition 3.6 which shows that a change of intersection coordinate by 2 can be realized by multiplying with at most four simple loops and arcs of small Dehn-Thurston coordinates.

In the next section, similar calculation will be done for the 1-holed torus and the 4-holed sphere.

3.2. Let us fix a colored H-decomposition of the 3 -holed sphere $\Sigma_{0,3}$ whose boundary components are $\partial_{1}, \partial_{2}, \partial_{3}$. Let $R$ be the red-hexagon. We assume that the cyclic order of $\left(\partial_{1} \cap R, \partial_{2} \cap R, \partial_{3} \cap R\right)$ coincides with the induced orientation on the boundary $\partial R$. We call $R \cap \partial_{i}$ the red arc in the boundary component. As a convention, in all figures, the red-hexagon is always drawn on the front face with the right-hand orientation.

Let us introduce some notations. Let $a_{r s}$ be a connected standard curve system (an arc) in $\Sigma_{0,3}$ so that it joins the $\partial_{r}$ and $\partial_{s}$, i.e., $I\left(a_{r s}, \partial_{k}\right)=$ $\delta_{r k}+\delta_{s k}$. Let $b_{i i}=\partial_{I} a_{i i}$ be the multiplication of the curve systems $\partial_{i}$ and $a_{i i}$. We will always assume that the intersection numbers $\left|a_{r s} \cap a_{u v}\right|$ and $\left|a_{r s} \cap b_{i i}\right|$ are minimal within their isotopy classes. Note that $\left|a_{i i} \cap a_{k k}\right|=2$ and $\left|a_{i i} \cap a_{j k}\right|=1$ for $i, j, k$ distinct, and all other intersection numbers are zero.

As a convention, we will always assume that when calculating the multiplication of two curve systems $a, b$ on a surface, $I(a, b)=|a \cap b|$.

3.3. We will find the explicit formula for the intersection number $I(\alpha, \beta)$ using the Dehn-Thurston coordinates of $\alpha, \beta$ in $C S\left(\Sigma_{0,3}\right)$. To this end, given a curve system $\alpha$ in $C S\left(\Sigma_{0,3}\right)$, let $n_{r s}(\alpha)$ be the number of components of $\alpha$ which are $\left[a_{r s}\right]$. Since $\alpha=\Pi_{r, s}\left[a_{r s}^{n_{r s}}\right]$ and the complete information on the intersections between $a_{i j}, a_{r s}$ is known, one finds the following formula,

$I(\alpha, \beta)=\sum_{(i, j, k) \in \mathcal{I}} n_{i i}(\alpha) n_{j k}(\beta)+n_{j k}(\alpha) n_{i i}(\beta)+2 n_{i i}(\alpha) n_{j j}(\beta)+2 n_{j j}(\alpha) n_{i i}(\beta)$,

where the index set $\mathcal{I}=\{(1,2,3),(2,3,1),(3,1,2)\}$.

In particular we obtain,

$$
I\left(\alpha, a_{11}\right)=n_{23}(\alpha)+2 n_{22}(\alpha)+2 n_{33}(\alpha),
$$

and

$$
I\left(\alpha, a_{23}\right)=n_{11}(\alpha)
$$



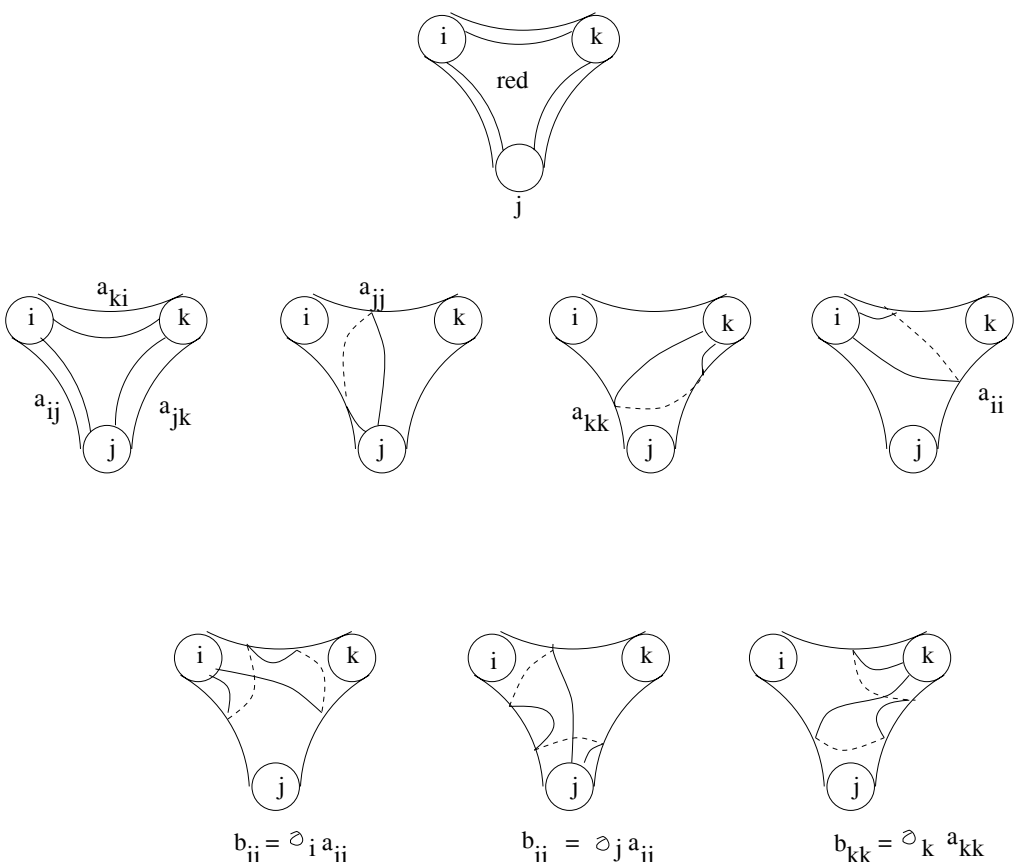

$b_{\text {ii }}=\partial_{i} a_{i i}$

$$
b_{j j}=\partial_{j} a_{j j}
$$

$\mathrm{b}_{\mathrm{kk}}={ }^{\partial} \mathrm{k} \mathrm{a}_{\mathrm{kk}}$

Right-hand orientation

Figure 3.1

Furthermore, if the Dehn-Thurston coordinate of $\alpha$ is $\left(x_{1}, x_{2}, x_{3}\right)$, then

$$
I\left(\alpha, a_{11}\right) \leq \max \left(x_{2}, x_{3}\right) \quad \text { and } \quad I\left(\alpha, a_{23}\right) \leq x_{1} / 2 .
$$

It remains to express the quantity $n_{r s}$ in terms of the Dehn-Thurston coordinate.

Lemma. There exist continuous homogeneous functions $f_{r s}\left(x_{1}, x_{2}, x_{3}\right)$ : $\mathbf{R}_{\geq 0}^{3} \rightarrow \mathbf{R}$ so that for all $\alpha$ in $C S\left(\Sigma_{0,3}\right)$ with Dehn-Thurston coordinate $\left(x_{1}, x_{2}, x_{3}\right), n_{r s}(\alpha)=f_{r s}\left(x_{1}, x_{2}, x_{3}\right)$.

Proof. Indeed, by symmetry, it suffices to write down the functions $f_{11}$ and $f_{23}$. By figure 3.1 and the classification of curve systems on the 3 -holed sphere, we see that

$$
f_{11}\left(x_{1}, x_{2}, x_{3}\right)=\frac{1}{2} \max \left(0, x_{1}-x_{2}-x_{3}\right)
$$

and

$$
f_{23}\left(x_{1}, x_{2}, x_{3}\right)= \begin{cases}\frac{1}{2} \max \left(0, x_{2}+x_{3}-x_{1}\right) & \text { if } x_{2}+x_{3} \geq x_{1} \\ \min \left(x_{2}, x_{3}\right) & \text { if } x_{2} \geq x_{1}+x_{3} \text { or } x_{3} \geq x_{1}+x_{2} \\ 0 & \text { otherwise }\end{cases}
$$


One checks easily that $f_{11}$ and $f_{23}$ are continuous and homogeneous functions defined on $\mathbf{R}^{3}$. QED

In particular, we see that in terms of the Dehn-Thurston coordinate $x=\left(x_{1}, x_{2}, x_{3}\right)$, the intersection pairing on $C S\left(\Sigma_{0,3}\right)$ is given by

$$
I(x, y)=\sum_{(i, j, k) \in \mathcal{I}} f_{i i}(x) f_{j k}(y)+f_{j k}(x) f_{i i}(y)+2 f_{i i}(x) f_{j j}(y)+2 f_{j j}(x) f_{i i}(y)
$$

Note that the map $I(x, y)$ is a continuous homogeneous function of real variables $\left(x_{1}, x_{2}, x_{3}, y_{1}, y_{2}, y_{3}\right) \in \mathbf{R}_{\geq 0}^{6}$. One of the goal of Thurston's theory is to show that the similar theorem holds for all surfaces.

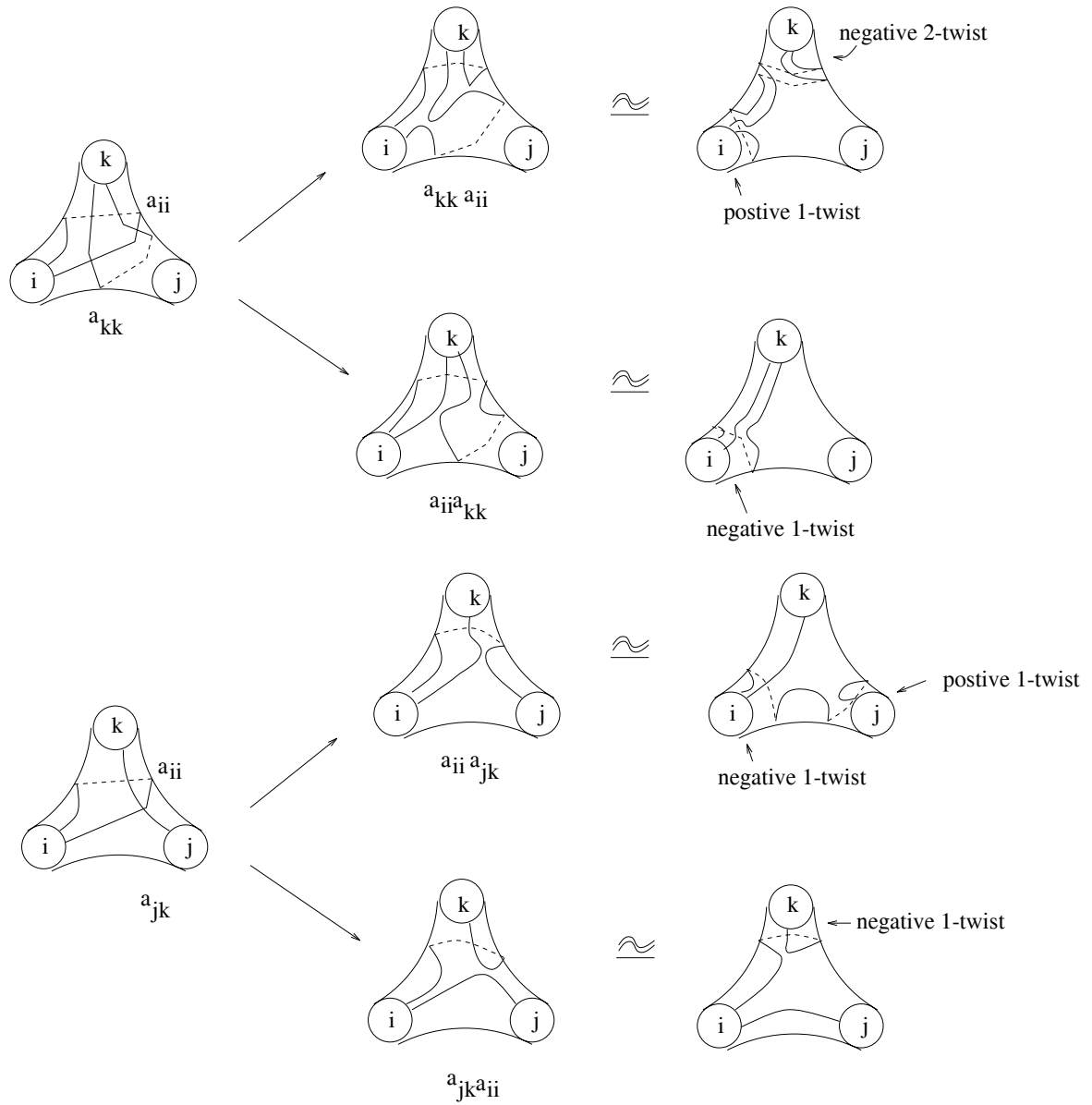

Figure 3.2

3.4. For $\alpha, \beta \in C S\left(\Sigma_{0,3}\right)$, we have $I\left(\alpha \beta, \partial_{i}\right)=I\left(\alpha, \partial_{i}\right)+I\left(\beta, \partial_{i}\right)$. This 
shows that

Lemma. Let $\Pi: C S(\Sigma) \rightarrow \mathbf{Z}^{3}$ be the Dehn-Thurston coordinate. Then $\Pi(\alpha \beta)=\Pi(\alpha)+\Pi(\beta)$.

In particular, we see that in $C S\left(\Sigma_{0,3}\right), \alpha \beta=\beta \alpha,\left[a_{i i} a_{j j}\right]=\left[a_{i j}^{2}\right]$, and $\left[a_{i i} a_{j k}\right]=\left[a_{i k} a_{i j}\right]$.

3.5. The multiplication $C S^{g}\left(\Sigma_{0,3}\right) \times C S^{g}\left(\Sigma_{0,3}\right) \rightarrow C S^{g}\left(\Sigma_{0,3}\right)$ is much more complicated. For instance, $a_{33} a_{11} \cong \partial_{1}\left(a_{13}^{2}\right) \partial_{3}^{2}$ in $C S^{g}\left(\Sigma_{0,3}\right)$. This extra twists along the boundary components is the main difficulty in proving theorem 1.1.

Below is the complete list of multiplications of $\alpha \in C S^{g}\left(\Sigma_{0,3}\right)$ having zero twisting coordinate with $\left[a_{r s}\right]$ so that $I\left(\alpha, a_{r s}\right) \neq 0$. (The case that $I\left(\alpha, a_{r s}\right)=0$, the multiplication is the disjoint union.) The basic identities underlying the proposition are the following four: $a_{i i} a_{k k}=a_{i k}^{2} \partial_{i}$, $a_{k k} a_{i i}=\partial_{i} a_{i k}^{2} \partial_{k}^{2}, a_{i i} a_{j k}=\partial_{j} a_{i j} a_{i k} \partial_{i}$, and $a_{j k} a_{i i}=a_{i j} a_{i k} \partial_{k}$ where $(i, j, k) \in$ $\{(1,2,3),(2,3,1),(3,1,2)\}$. See figure 3.2 for a verification.

Proposition. Suppose $(i, j, k) \in\{(1,2,3),(2,3,1),(3,1,2)\}$. Then the following identities hold where isotopies leave red-arcs in the boundary components invariant. We assume that the index $l>0$.

$$
\left(a_{i k}^{n} a_{j k}^{m} a_{k k}^{l}\right) a_{i i} \cong \partial_{i}\left(a_{i k}^{n+2} a_{j k}^{m} a_{k k}^{l-1}\right) \partial_{k}^{2}
$$

$$
a_{i i}\left(a_{i k}^{n} a_{j k}^{m} a_{k k}^{l}\right) \cong\left(a_{i k}^{n+2} a_{j k}^{m} a_{k k}^{l-1}\right) \partial_{i}
$$

$$
\left(a_{i j}^{n} a_{i k}^{m} a_{k j}^{l}\right) a_{i i} \cong\left(a_{i j}^{n+1} a_{i k}^{m+1} a_{k j}^{l-1}\right) \partial_{k}
$$

$$
a_{i i}\left(a_{i j}^{n} a_{j k}^{m} a_{k j}^{l}\right) \cong \partial_{j}\left(a_{i j}^{n+1} a_{i k}^{m+1} a_{k j}^{l-1}\right) \partial_{i},
$$

$$
\left(a_{i j}^{n} a_{j k}^{m} a_{j j}^{l}\right) a_{i i} \cong\left(a_{i j}^{n+2} a_{j k}^{m} a_{j j}^{l-1}\right) \partial_{j},
$$

$$
a_{i i}\left(a_{i j}^{n} a_{j k}^{m} a_{j j}^{l}\right) \cong \partial_{j}\left(a_{i j}^{n+2} a_{j k}^{m} a_{j j}^{l-1}\right) \partial_{i}^{2}
$$

$$
\left(a_{i k}^{n} a_{j k}^{m} a_{k k}^{l}\right) a_{i j} \cong \partial_{i}\left(a_{i k}^{n+1} a_{j k}^{m+1} a_{k k}^{l-1}\right) \partial_{k},
$$

$$
a_{i j}\left(a_{i k}^{n} a_{j k}^{m} a_{k k}^{l}\right) \cong\left(a_{i k}^{n+1} a_{j k}^{m+1} a_{k k}^{l-1}\right) \partial_{j} .
$$

Proof. The proof follows from the figures below. 

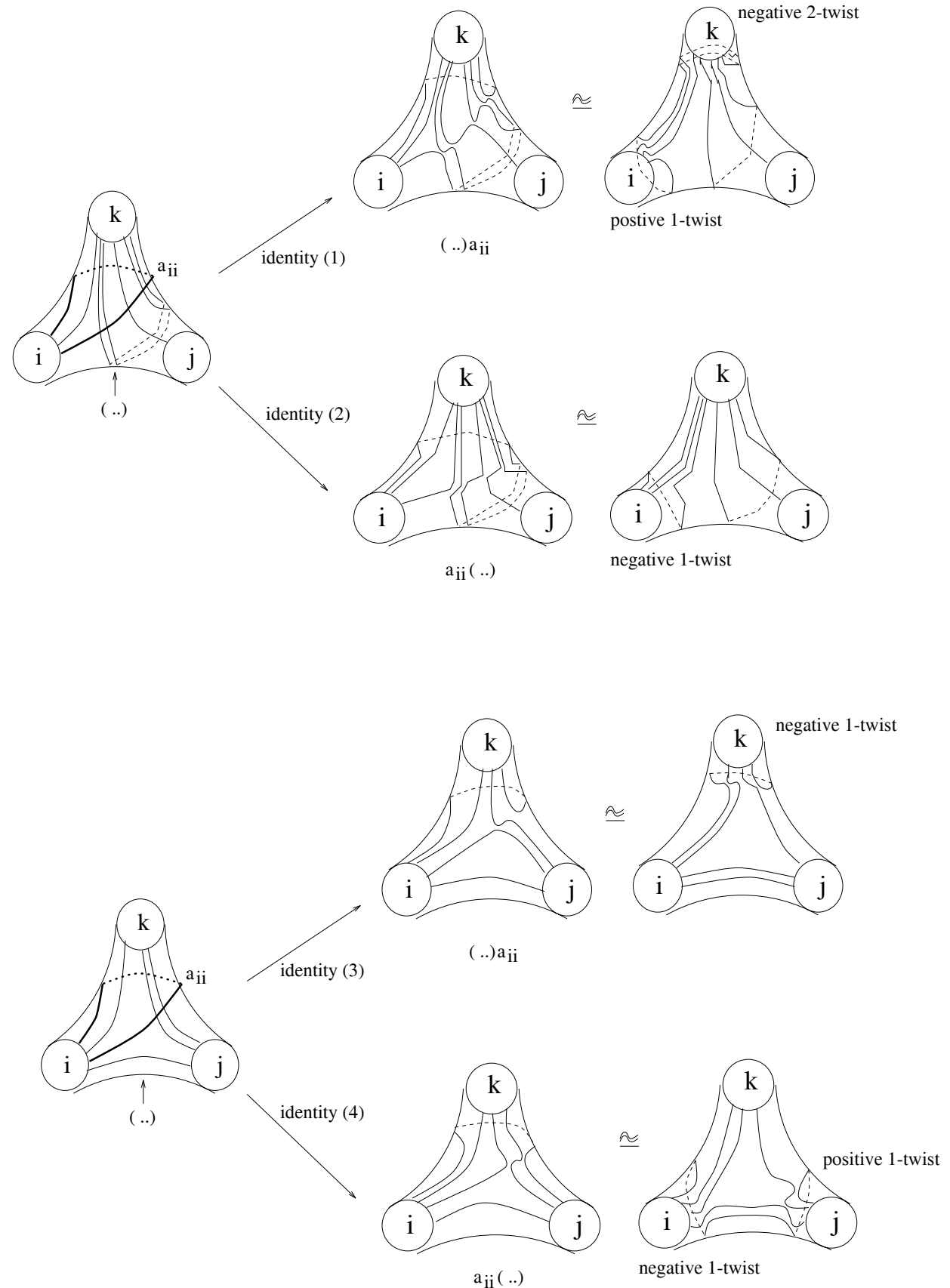

Figure 3.3 

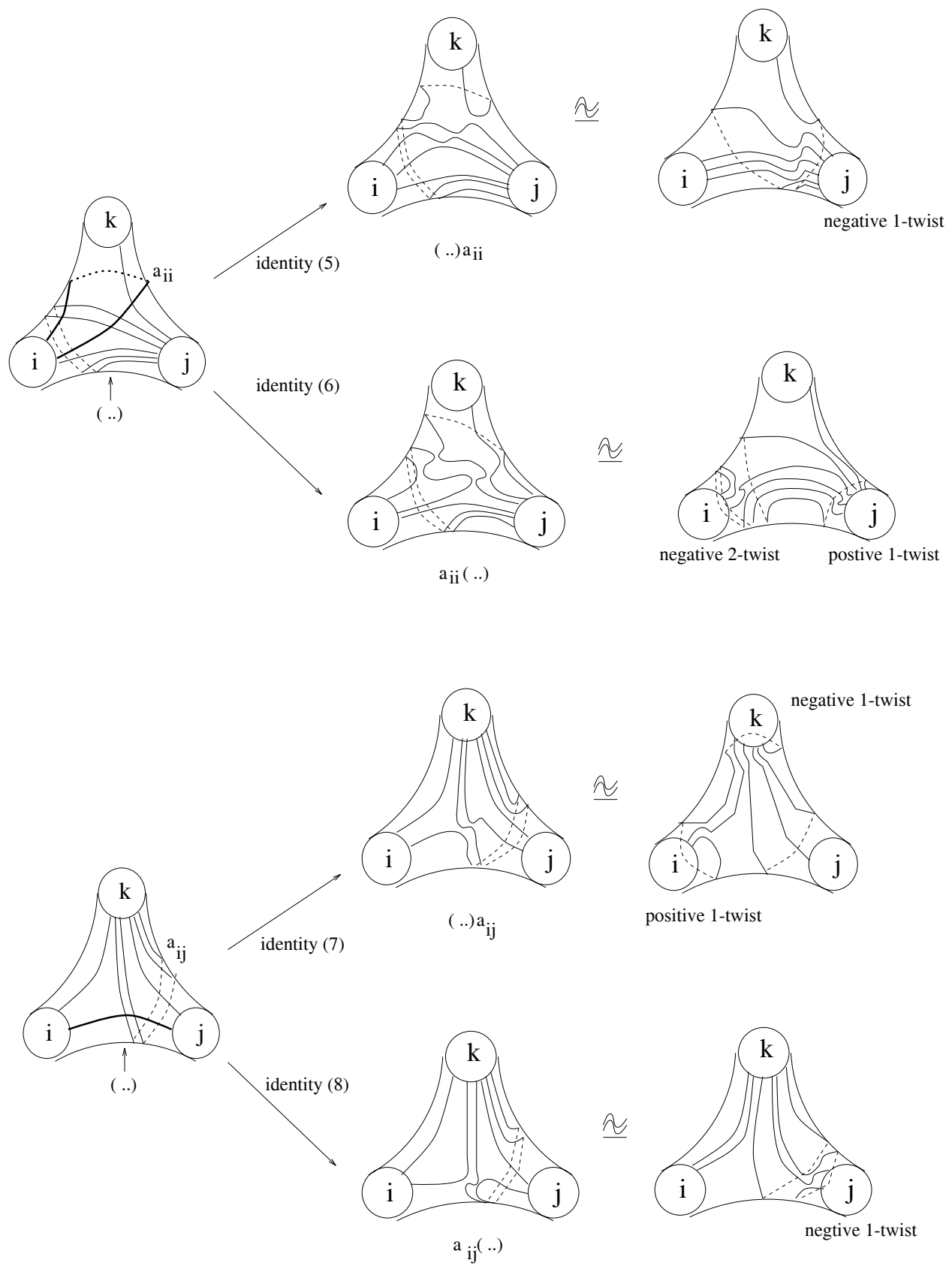

Figure 3.4

Note that the exponents of $a_{i j}$ 's in the above formula are governed by lemma 3.4. By combining lemma 3.4 with the associativity of the multipli- 
cations (theorem 2.4), this proposition gives the complete information about the multiplication between two classes in $C S^{g}\left(\Sigma_{0,3}\right)$. However, we do not know a simple expression like lemma 3.4 for the multiplication in $C S^{g}\left(\Sigma_{0,3}\right)$.

For applications, we will need the following additional formulas. Recall that $b_{i i}=\partial_{i} a_{i i}$.

Corollary. Under the same assumption as in the proposition, the following identities hold.

(1)

$$
\left(a_{i k}^{n} a_{j k}^{m} a_{k k}^{l}\right)\left(a_{i j} \partial_{i}\right) \cong\left(a_{i k}^{n+1} a_{j k}^{m+1} a_{k k}^{l-1}\right) \partial_{k},
$$

$$
\left(\partial_{j} a_{i j}\right)\left(a_{i k}^{n} a_{j k}^{m} a_{k k}^{l}\right) \cong a_{i k}^{n+1} a_{j k}^{m+1} a_{k k}^{l-1}
$$

$$
\left(a_{i k}^{n} a_{j k}^{m} a_{k k}^{l}\right)\left(a_{i i} \partial_{i}\right) \cong\left(a_{i k}^{n+2} a_{j k}^{m} a_{k k}^{l-1}\right) \partial_{k}^{2}
$$

$$
\begin{gathered}
b_{i i}\left(a_{i k}^{n} a_{j k}^{m} a_{k k}^{l}\right) \cong a_{i k}^{n+2} a_{j k}^{m} a_{k k}^{l-1}, \\
b_{i i}\left(a_{i j}^{n} a_{j k}^{m} a_{j j}^{l}\right) \cong \partial_{j}\left(a_{i j}^{n+1} a_{j k}^{m+1} a_{k j}^{l-1}\right) \partial_{i},
\end{gathered}
$$

$$
\left(\partial_{i} b_{i i}\right)\left(a_{i j}^{n} a_{j k}^{m} a_{j j}^{l}\right) \cong \partial_{j}\left(a_{i j}^{n+2} a_{j k}^{m} a_{j j}^{l-1}\right),
$$

Indeed, the corollary follows from theorem 2.4(4) and the identities in the previous proposition. Indeed, by theorem 2.4(4), if $\alpha$ intersects $\partial_{i}$ and $\beta=\alpha \partial_{i}^{n}$, then $\alpha=\partial_{i}^{n} \beta$. Thus in the corollary above, identity (1) follows from proposition 3.5(7); identity (2) follows from 3.5(8); identity (3) follows from 3.5(1); identity (4) follows from 3.5(1); identity (5) follows from 3.5(6); and identity (6) follows from 3.5(6).

The following proposition is the main result in the section. It shows that if the Dehn-Thurston coordinate is changed by 2, then the corresponding curve systems under go a multiplication by at most four simple curve systems of small Dehn-Thurston coordinates.

3.6. Proposition. Suppose the Dehn-Thurston coordinate $\left(\left(x_{1}, 0\right),\left(x_{2}, 0\right)\right.$, $\left.\left(x_{3}, 0\right)\right)$ is represented by $\gamma \in C S^{g}\left(\Sigma_{0,3}\right)$ where $x_{i}=I\left(\gamma, \partial_{i}\right)$. Then,

(1) the Dehn-Thurston coordinate $\left(\left(x_{1}+2,0\right),\left(x_{2}, 0\right),\left(x_{3}, 0\right)\right)$ is represented by $\left(\gamma\left[a_{11}\right] \partial_{1}^{e_{1}}\right) \partial_{2}^{e_{2}} \partial_{3}^{e_{3}}$ and by $\left(\partial_{1}^{e_{1}}\left[b_{11}\right] \gamma\right) \partial_{2}^{e_{2}} \partial_{3}^{e_{3}}$ where $e_{1} \in\{0,1\}$ and $\left|e_{2}\right|+\left|e_{3}\right| \leq 2$.

(2) the Dehn-Thurston coordinate $\left(\left(x_{1}+1,0\right),\left(x_{2}, 0\right),\left(x_{3}+1,0\right)\right)$ is represented by $\left(\gamma\left[a_{13}\right] \partial_{1}\right) \partial_{2}^{e_{2}}$ and by $\left(\partial_{3}\left[a_{13}\right] \gamma\right) \partial_{2}^{e_{2}}$ where $\left|e_{2}\right| \leq 2$. 
Proof. Take a standard curve system $c$ in $\gamma$. To see part (1), we will calculate the Dehn-Thurston coordinate of $\left[a_{11}\right] \gamma$ and $\gamma\left[b_{11}\right]$. For simplicity, we just calculate $\left[a_{11}\right] \gamma$. If $I\left(\gamma, a_{11}\right)=0$, then clearly $\gamma\left[a_{11}\right]$ has the DehnThurston coordinate $\left(\left(x_{1}+2,0\right),\left(x_{2}, 0\right),\left(x_{3}, 0\right)\right)$. If $I\left(\gamma, a_{11}\right)>0$, then $\gamma=$ $a_{13}^{m} a_{23}^{n} a_{33}^{l}$, or $a_{12}^{m} a_{23}^{m} a_{22}^{l}$ or $a_{12}^{m} a_{13}^{n} a_{23}^{l}$ where $l>0$. Now we use the fact that if $\alpha$ intersects $\partial_{i}$ and $\beta=\alpha \partial_{i}^{n}$, then $\alpha=\partial_{i}^{n} \beta$ (theorem 2.4(4)). Thus the result follows from proposition $3.5(3),(5)$ and corollary $3.5(3)$ for $(i, j, k)=$ $(1,2,3)$. The multiplication by $\partial_{1}^{s} b_{11}$ follows from corollary $3.5(4),(6)$ and proposition 3.5(4). Finally part (2) follows from corollary $3.5(1),(2)$ for $(i, j, k)=(3,1,2)$.

\section{Calculations on the 1-holed Torus and the 4-holed Sphere.}

4.1. The goal of this section is to show that the change of Dehn-Thurston coordinates can be achieved by multiplying curve systems by some simple loops on the 1-holed torus and the 4-holed sphere. To be more precise, given a curve system $\alpha \in C S^{g}(\Sigma)$, if the $i$-th intersection coordinate of $\alpha$ is changed by 2 , we will prove in this section that the change can be achieved by multiplying $\alpha$ by at most five simple loops whose Dehn-Thurston coordinates are small.

In the last part of this section, we prove that the $i$-th Dehn-Thurston coordinate can be expressed as a continuous homogeneous function on geometric intersection numbers with a fixed set of simple loops.

In this section, we take as our convention that all surfaces have a colored $\mathrm{H}$-decomposition so that the red-hexagons are drawn on the front face with the right-hand orientation.

4.2. Take a colored H-decomposition $\left(\left[p_{1}\right] ;\left[c_{1} \cup c_{2}\right]\right)$ of the 1-holed torus. Let $p_{2}=\partial \Sigma_{1,1}$. See figure 4.1 .

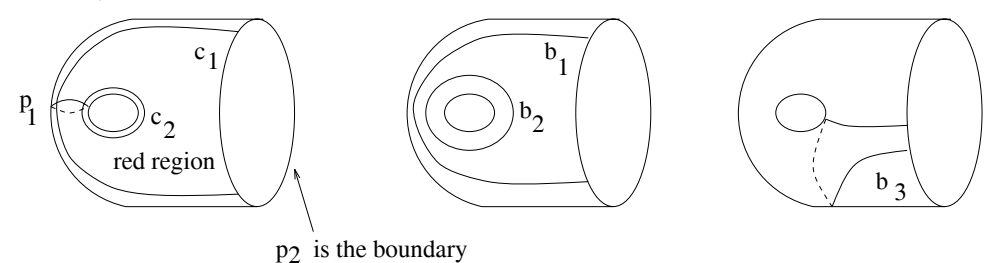

Right-hand orientation on the red region

Figure 4.1

Proposition. Suppose the Dehn-Thurston coordinate $\left(\left[x_{1}, t_{1}\right],\left(x_{2}, 0\right)\right)$ is represented by $\gamma \in C S^{g}\left(\Sigma_{1,1}\right)$. Then the Dehn-Thurston coordinate $\left(\left[x_{1}+\right.\right.$ 
$\left.\left.1, t_{1}\right],\left(x_{2}, 0\right)\right)$ is represented by the class $\left[p_{2}\right]^{e_{2}}\left(\delta^{e_{1}} \gamma\right)$ where $\delta$ is represented by a simple loop and $e_{1} \in\{ \pm 1\}$ and $e_{2} \in\{0, \pm 1\}$. Furthermore, for all $\alpha \in C S^{g}\left(\Sigma_{1,1}\right), I(\alpha, \delta)+I\left(\alpha, p_{2}\right) \leq 2|\Pi(\alpha)|$.

Proof. We write $\gamma=\left[p_{1}\right]^{t_{1}} \gamma_{z t}$ where $\gamma_{z t}$ has zero-twisting coordinate. Take a curve system $c$ in $\gamma_{z t}$ and let $b_{1}, b_{2}, b_{3}$ be the good curve systems in $\Sigma_{1,1}$ so that their Dehn-Thurston coordinates are $([1,0],(2,0)),([1,0],(0,0))$ and $([0,0],(2,0))$ as shown in figure 4.1. By definition, either $c \cong b_{1}^{n_{1}} b_{2}^{n_{2}}$ or $c \cong b_{1}^{n_{1}} b_{3}^{n_{3}}$ where $n_{3}>0$. If $c \cong b_{1}^{n_{1}} b_{2}^{n_{2}}$, then we take $\delta=\left[b_{2}\right]$ and $e_{1}=1$, $e_{2}=0$. Thus depending on the sign of the twisting coordinate $t_{1}$, either $\delta \gamma$ or $\gamma \delta$ has the Dehn-Thurston coordinate of the required form.

If $c \cong b_{1}^{n_{1}} b_{3}^{n_{3}}$, let $\Sigma^{\prime}$ be the 3 -holed sphere $\Sigma_{1,1}-i n t\left(N\left(p_{1}\right)\right)$ where $N\left(p_{1}\right)$ is a small regular neighborhood of $p_{1}$. We give $\Sigma^{\prime}$ the restriction orientation and the restriction colored $\mathrm{H}$-decomposition. The boundary components of $\Sigma^{\prime}$ are labeled as $\partial_{1}, \partial_{2}, \partial_{3}$ where $\partial N\left(p_{1}\right)=\partial_{1} \cup \partial_{3}$ so that the cyclic order $\left(\partial_{1}, \partial_{2}, \partial_{3}\right)$ coincides with the induced orientation on the boundary of the red-hexagon in $\Sigma^{\prime}$. Let $a_{13}=b_{2} \cap \Sigma^{\prime}$.

The choice of the loop $\delta$ depends on the sign of the twisting coordinate $t_{1}=t_{1}(\gamma)$. If $t_{1} \geq 0$, we choose $\delta=\left[b_{2} p_{1}\right]$. Since the Dehn-Thurston coordinate of $c \cap \Sigma^{\prime}$ in $C S^{g}\left(\Sigma^{\prime}\right)$ is $\left(\left(x_{1}, 0\right),\left(x_{2}, 0\right),\left(x_{1}, 0\right)\right)$, by proposition 3.6 , the Dehn-Thurston coordinate of $\left(c \cap \Sigma^{\prime}\right)\left(a_{13} \partial_{1}\right)$ is $\left(\left(x_{1}+1,0\right),\left(x_{2},-1\right),\left(x_{1}+\right.\right.$ $1,0))$. Note that the twisting coordinates of $c \cap \Sigma^{\prime}$ at the $\partial_{1}$-th and $\partial_{3}$-th coordinate are zero. Combining this with the fact that $\left|c \cap\left(b_{2} \partial_{1}\right)\right|=I(c, \delta)$ and $\mid\left(c \cap\left(b_{2} \partial_{1}\right) \cap N\left(p_{1}\right) \mid=0\right.$, we see that the Dehn-Thurston coordinate of $\gamma_{z t} \delta$ is $\left(\left[x_{1}+1,0\right],\left(x_{2},-1\right)\right)$. On the other hand, by figure 4.2 , we see that the triple $\left(p_{1}^{t_{1}}, \gamma_{z t}, \delta\right)$ contains no quadrilaterals and only negative triangles. Thus by the associativity for the multiplication (theorem 2.4), $\gamma \delta=\left[p_{1}^{t_{1}}\right]\left(\delta_{z t} \delta\right)$. This shows that the Dehn-Thurston coordinate of $\gamma \delta$ is of the form $\left(\left[x_{1}+1, t_{1}\right],\left(x_{2},-1\right)\right)$. Thus the Dehn-Thurston coordinate of $\gamma \delta\left[p_{2}\right]^{-1}$ has the required form.

If the twisting coordinate $t_{1}(\gamma)<0$, we take $\delta=\left[p_{1} b_{2}\right]=\left[\partial_{1} b_{2}\right]$ and consider $\delta \gamma$. The proof is the same as above and the only place we need to pay attention is that the triple $\left(\gamma_{z t}, p_{1}^{-t_{1}}, \delta\right)$ contains no positive triangles and positive quadrilaterals.

To estimate the intersection number $I(\alpha, \delta)+I\left(\alpha, p_{2}\right)$ for any curve system $\alpha \in C S^{g}\left(\Sigma_{1,1}\right)$, take $a \in \alpha$ so that $a$ intersects $b_{2}, p_{1}$ and $p_{2}$ minimally. Note that by the triangle inequality (theorem 2.4(6)) $I(\alpha, \delta)+I\left(\alpha, p_{2}\right) \leq$ $I\left(\alpha, p_{1}\right)+I\left(\alpha, b_{2}\right)+I\left(\alpha, p_{2}\right)=x_{1}(\alpha)+\left|a \cap b_{2}\right|+x_{2}(\alpha)$. Now since $a=p_{1}^{t} a_{z t}$ where $a_{z t}$ is a standard zero twisting representative of $\alpha_{z t}$, we see that $\left|a \cap b_{2}\right|=\left|\left(p_{1}^{t_{1}} a_{z t}\right) \cap b_{2}\right| \leq\left|p_{1}^{\left|t_{1}\right|} \cap b_{2}\right|+\left|a_{z t} \cap b_{2}\right| \leq\left|t_{1}\right|+\left|a_{z t} \cap b_{2}\right|$. But 
the intersection $a_{z t} \cap b_{2}$ is inside the 3-holed sphere $\Sigma^{\prime}$ and the intersection number $I\left(a_{z t}, b_{2}\right)$ is equal to the intersection number $I\left(a_{z t} \cap \Sigma^{\prime}, b_{2} \cap \Sigma^{\prime}\right)$ by the choice of the standard curve systems. Thus by inequality (3.4), $\left|a_{z t} \cap b_{2}\right| \leq \frac{1}{2} I\left(\alpha_{z t}, \partial_{2}\right)=\frac{1}{2} x_{2}(\alpha)$. In summary, we obtain $I(\alpha, \delta)+I\left(\alpha, p_{2}\right) \leq$ $3 / 2\left(x_{1}(\alpha)+x_{2}(\alpha)+\left|t_{1}\right|\right)=3 / 2|\Pi(\alpha)| \leq 2|\Pi(\alpha)|$.
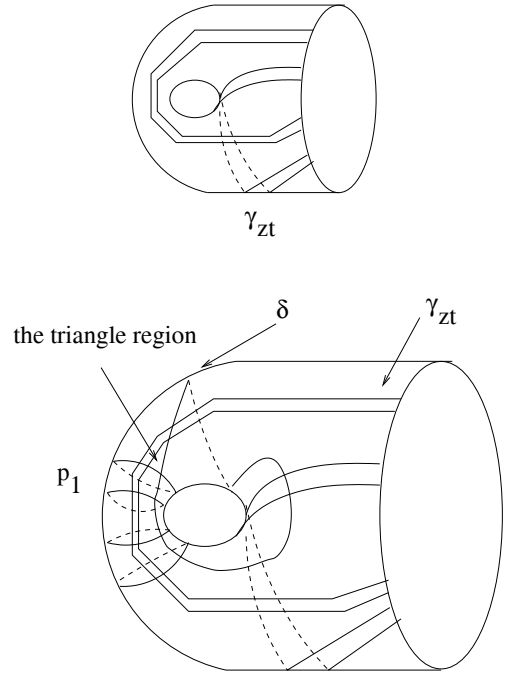
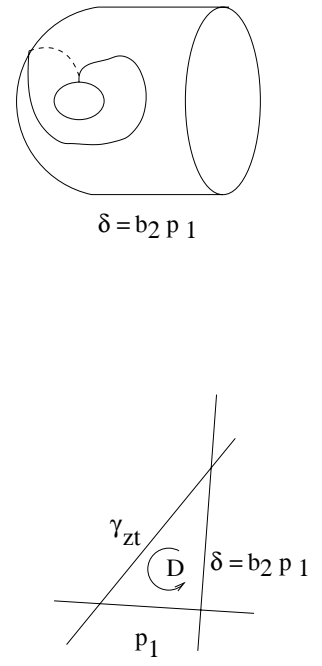

Figure 4.2

Remark. The crucial fact that the intersection number of two standard zero-twisting curve systems in $\Sigma_{1,1}$ is equal to the intersection number of their restrictions to the 3 -holed sphere is no longer valid for the 4-holed sphere. One needs a more careful analysis of the intersection number in the later case.

4.3. Take a colored H-decomposition $\left(\left[p_{1}\right],\left[d_{1} \cup \ldots \cup d_{4}\right]\right)$ of the oriented 4-holed sphere $\Sigma_{0,4}$ whose boundary components are $\partial_{i}$ for $i=2,3,2^{\prime}, 3^{\prime}$. Let $p_{i}=\partial_{i}$ for $i=2,3,2^{\prime}, 3^{\prime}$. See figure 1.1 .

Proposition. Suppose that the Dehn-Thurston coordinate $\left(\left[x_{1}, t_{1}\right],\left(x_{2}, 0\right)\right.$, $\left.\left(x_{3}, 0\right),\left(x_{2^{\prime}}, 0\right),\left(x_{3^{\prime}}, 0\right)\right)$ is represented by $\gamma \in C S^{g}\left(\Sigma_{0,4}\right)$. Then the DehnThurston coordinate $\left(\left[x_{1}+2, t_{1}\right],\left(x_{2}, 0\right),\left(x_{3}, 0\right),\left(x_{2^{\prime}}, 0\right),\left(x_{3^{\prime}}, 0\right)\right)$ is represented by the class $p_{2}^{s_{2}} p_{3}^{s_{3}} p_{2^{\prime}}^{s_{2^{\prime}}} p_{3^{\prime}}^{s_{3^{\prime}}}\left(\delta^{e} \gamma\right)$ where $\delta$ is represented by a simple loop, $e \in$ $\{ \pm 1\}$, and $\left|s_{2}\right|+\left|s_{3}\right|$ and $\left|s_{2^{\prime}}\right|+\left|s_{3^{\prime}}\right|$ are both at most 2. Furthermore, for all $\alpha \in C S(\Sigma)$, the following inequalities hold. 
(1)

$$
I(\alpha, \delta)+I\left(\alpha, p_{2}^{\left|s_{2}\right|} p_{3}^{\left|s_{3}\right|} p_{2}^{\left|s_{2^{\prime}}\right|} p_{3^{\prime}}^{\left|s_{3^{\prime}}\right|}\right) \leq 4|\Pi(\alpha)| .
$$

(2) for all partitions of $\left\{2,2^{\prime}, 3,3^{\prime}\right\}$ into a 2-element set $\{i, j\} \cup\{k, l\}$,

$$
\begin{aligned}
& I(\alpha, \delta)+\left(\left|s_{i}\right|+\left|s_{j}\right|\right) \max \left(I\left(\alpha, p_{i}\right), I\left(\alpha, p_{j}\right)\right)+\left(\left|s_{k}\right|+\left|s_{l}\right|\right) \max \left(I\left(\alpha, p_{k}\right), I\left(\alpha, p_{l}\right)\right) \\
& \quad \leq 4 x_{1}(\alpha)+2\left|t_{1}(\alpha)\right|+6 \max \left(I\left(\alpha, p_{i}\right), I\left(\alpha, p_{j}\right)\right)+6 \max \left(I\left(\alpha, p_{k}\right), I\left(\alpha, p_{l}\right)\right) .
\end{aligned}
$$

(3) if in addition to (2) we assume that $\{i, j\}=\{2,3\}$ and $\{k, l\}=\left\{2^{\prime}, 3^{\prime}\right\}$, then

$$
\begin{aligned}
I(\alpha, \delta)+\left(\left|s_{2}\right|+\left|s_{3}\right|\right) \max ( & \left.I\left(\alpha, p_{2}\right), I\left(\alpha, p_{3}\right)\right) \\
+\left(\left|s_{2^{\prime}}\right|\right. & \left.+\left|s_{3^{\prime}}\right|\right) \max \left(I\left(\alpha, p_{2^{\prime}}\right), I\left(\alpha, p_{3^{\prime}}\right)\right) \\
\leq 4 x_{1}(\alpha)+2\left|t_{1}(\alpha)\right|+4 \max ( & \left.I\left(\alpha, p_{2}\right), I\left(\alpha, p_{3}\right)\right) \\
& +4 \max \left(I\left(\alpha, p_{2^{\prime}}\right), I\left(\alpha, p_{3^{\prime}}\right)\right) .
\end{aligned}
$$

Proof. Let us write $\gamma=\left[p_{1}^{t_{1}}\right] \gamma_{z t}$ where $\gamma_{z t}$ has zero-twisting coordinate. Let $a_{11}$ be a standard curve system in $\Sigma_{0,4}$ whose Dehn-Thurston coordinate is $([2,0],(0,0),(0,0),(0,0),(0,0))$. Let $b_{11}=p_{1}^{2} a_{11}$. See figure 4.3 .
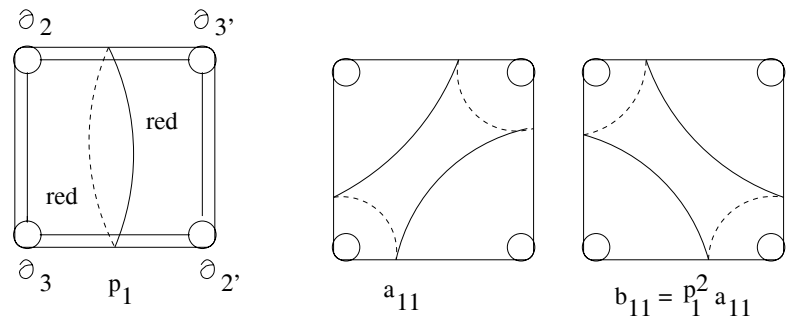

Figure 4.3

The required class $\delta$ will be either $\left[a_{11} p_{1}^{s}\right]$ or $\left[p_{1}^{s} b_{11}\right]$ where $s \in\{0,1,2\}$ depending on the sign of the twisting coordinate $t_{1}(\gamma)$. Using theorem 2.4(4), we will show that the Dehn-Thurston coordinate of the class $\delta^{e} \gamma$ for appropriate choices of $e$ has the form $\left(\left[x_{1}, t_{1}\right],\left(x_{2},-s_{2}\right),\left(x_{3},-s_{3}\right),\left(x_{2^{\prime}}, s_{2^{\prime}}\right),\left(x_{3^{\prime}}, s_{3^{\prime}}\right)\right)$.

The basic fact that we need is in the following lemma.

Lemma. Suppose $\gamma_{z t}$ is a curve system in $C S^{g}\left(\Sigma_{0,4}\right)$ with Dehn-Thurston coordinate $\left(\left[x_{1}, 0\right],\left(x_{2}, 0\right),\left(x_{3}, 0\right),\left(x_{2^{\prime}}, 0\right),\left(x_{3^{\prime}}, 0\right)\right)$. Then

(1) for $n, s$ in $\mathbf{Z}_{\geq 0}$, the triples of curve systems $\left(\left[p_{1}^{n}\right], \gamma_{z t},\left[a_{11} p^{s}\right]\right)$ and $\left(\left[p_{1}^{s} b_{11}\right]\right.$, $\left.\gamma_{z t},\left[p_{1}\right]^{n}\right)$ do not contain positive triangles or positive quadrilaterals, 
(2)

$$
\begin{aligned}
I\left(\gamma_{z t}, a_{11}\right)=\left(f_{12}+2 f_{22}\right. & \left.+f_{23}+2 f_{33}\right)\left(x_{1}, x_{2}, x_{3}\right) \\
& +\left(f_{12}+2 f_{22}+f_{23}+2 f_{33}\right)\left(x_{1}, x_{2^{\prime}}, x_{3^{\prime}}\right),
\end{aligned}
$$

and

$$
\begin{aligned}
I\left(\gamma_{z t}, b_{11}\right)=\left(f_{11}+2 f_{22}\right. & \left.+2 f_{33}+f_{13}+f_{23}\right)\left(x_{1}, x_{2}, x_{3}\right) \\
+ & \left(f_{11}+2 f_{22}+2 f_{33}+f_{13}+f_{23}\right)\left(x_{1}, x_{2^{\prime}}, x_{3^{\prime}}\right) .
\end{aligned}
$$

$$
I\left(\gamma_{z t}, a_{11}\right) \leq \max \left(x_{2}, x_{3}\right)+\max \left(x_{2^{\prime}}, x_{3^{\prime}}\right)
$$

and

$$
I\left(\gamma_{z t}, b_{11}\right) \leq 2 x_{1}+\max \left(x_{2}, x_{3}\right)+\max \left(x_{2^{\prime}}, x_{3^{\prime}}\right) .
$$

Proof. We begin by introduce some notations. Let the two 3-holed sphere components of $\Sigma_{0,4}-\operatorname{int}\left(N\left(p_{1}\right)\right)$ be $\Sigma^{+}$and $\Sigma^{-}$. The boundary components of $\Sigma^{+}$and $\Sigma^{-}$are $\partial_{2}, \partial_{3}, \partial_{1}^{+}$and $\partial_{2^{\prime}}, \partial_{3^{\prime}}, \partial_{1}^{-}$respectively. The subsurfaces $\Sigma^{+}$and $\Sigma^{-}$have the induced orientations and the restriction colored $\mathrm{H}$ decompositions so that the cyclic orders $\left(\partial_{2}, \partial_{3}, \partial_{1}^{+}\right)$and $\left(\partial_{2^{\prime}}, \partial_{3^{\prime}}, \partial_{1}^{-}\right)$coincide with the orientations on the boundary of the red-hexagons. Choose a standard representative $c$ of $\gamma_{z t}$ so that all pairs $(c, x)$ have minimal intersection numbers within their isotopy classes for $x \in\left\{a_{11}, b_{11}, p_{1}\right\}$. Furthermore, all pairs $\left(c \cap \Sigma^{ \pm}, x \cap \Sigma^{ \pm}\right)$have the minimal intersection numbers within their isotopy classes in the subsurface $\Sigma^{ \pm}$. Let $Q$ be the red-rectangle in $N\left(p_{1}\right)$ which is the intersection of the annulus $N\left(p_{1}\right)$ with the red region. The two sides of $Q$ in $\partial N\left(p_{1}\right)$ are denoted by $I_{-}$and $I_{+}$so that $I_{ \pm} \subset \partial \Sigma^{ \pm}$and that $I_{ \pm}$have the induced orientation from $\Sigma^{ \pm}$. By the construction, we choose $c \in \gamma_{z t}$ so that $a_{11} \cap I_{ \pm}$contains the first point among the intersection points $\left(c \cup a_{11}\right) \cap I_{ \pm}$(measured in the orientation of $I_{ \pm}$.) Similarly, $b_{11} \cap I_{ \pm}$contains the last point among the intersection points $\left(c \cup b_{11}\right) \cap I_{ \pm}$. See figure 4.4.

Now to see part (1), we note that if we parameterize the annulus $N\left(p_{1}\right)$ by $S^{1} \times I$ so that all intersections $c \cap p_{1}$ and $\partial Q \cap i n t\left(N\left(p_{1}\right)\right)$ are "horizontal" of the form $p \times I$, then the $a_{11} \cap Q$ consists of two arcs joining the lower left corner (the first point of $I_{+}$) of $Q$ to the upper right corner (the first points in $\left.I_{-}\right)$of $Q$. This shows that the ordered triple $\left(\left[p_{1}^{n}\right], \gamma_{z t},\left[a_{11}\right]\right)$ contains no quadrilaterals and no positive triangles. It may contain some negatively oriented triangles as shown in figure 4.4.

If we replace $a_{11}$ by $a_{11} p_{1}^{s}$ where $s>0$, then $a_{11} p_{1}^{s} \cap N\left(p_{1}\right)$ has larger positive slope than that of $a_{11} \cap N\left(p_{1}\right)$. Thus the triple $\left(\left[p_{1}^{n}\right], \gamma_{z t},\left[a_{11} p_{1}^{s}\right]\right)$ 
again contains no positive triangles and quadrilaterals. The proof for the triple $\left(\left[p_{1}^{s} b_{11}\right], \gamma_{z t},\left[p_{1}\right]^{n}\right)$ is the same.
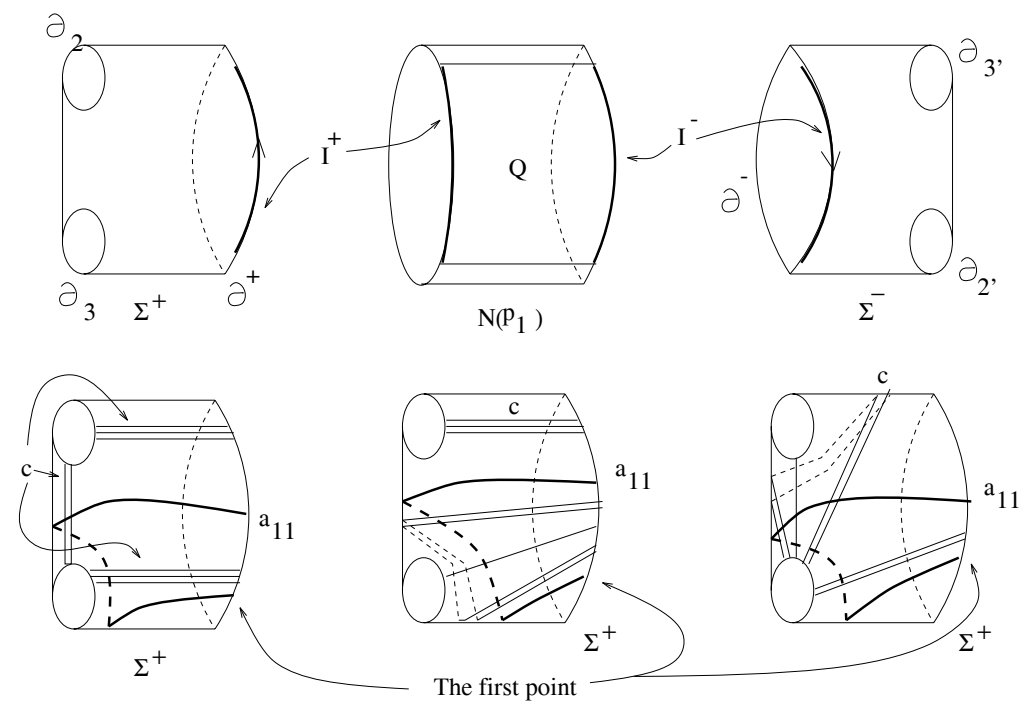

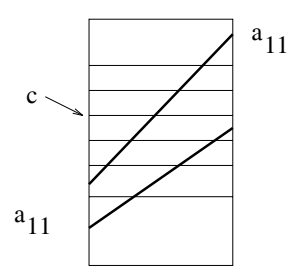

Q

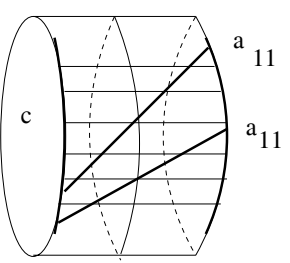

$\mathrm{p}_{1}$

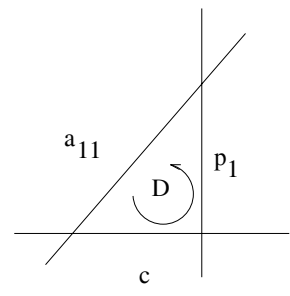

Figure 4.4

To see part (2), we note that $I\left(\gamma_{z t}, a_{11}\right)=\left|c \cap a_{11}\right|$ by construction. Now the intersection number $\left|c \cap a_{11}\right|$ is equal to the sum $\left|c \cap a_{11} \cap \Sigma^{+}\right|+\mid c \cap a_{11} \cap$ $\Sigma^{-}|+| c \cap a_{11} \cap N\left(p_{1}\right) \mid$. By the construction, $\left|c \cap a_{11} \cap \Sigma^{ \pm}\right|$is $I\left(c \cap \Sigma^{ \pm}, a_{11} \cap \Sigma^{ \pm}\right)$. By identity (3.2), we have $I\left(c \cap \Sigma^{+}, a_{11} \cap \Sigma^{+}\right)=\left(2 f_{22}+f_{23}+2 f_{33}\right)\left(x_{1}, x_{2}, x_{3}\right)$ and $I\left(c \cap \Sigma^{-}, a_{11} \cap \Sigma^{-}\right)=\left(2 f_{22}+f_{23}+2 f_{33}\right)\left(x_{1}, x_{2^{\prime}}, x_{3^{\prime}}\right)$. Now the intersection number $\left|c \cap a_{11} \cap N\left(p_{1}\right)\right|$ is the sum $\left(x_{1}-g_{+}\right)+\left(x_{1}-g_{-}\right)$where $g_{ \pm}$is the number of points of $c \cap I_{ \pm}$which is between $a_{11} \cap I_{ \pm}$in the interval $I_{ \pm}$. See figure 4.5 .

But by the construction of $c$ and $a_{11}, g_{ \pm}$is exactly the number $\left(n_{11}+\right.$ $\left.n_{31}\right)\left(\left[c \cap \Sigma^{ \pm}\right]\right)$, i.e., $x_{1}-g_{ \pm}=n_{12}\left(\left[c \cap \Sigma^{ \pm}\right]\right)$(see figure 4.5). Thus $x_{1}-g_{+}=$ $f_{12}\left(x_{1}, x_{2}, x_{3}\right)$ and $x_{1}-g_{-}=f_{12}\left(x_{1}, x_{2^{\prime}}, x_{3^{\prime}}\right)$. This implies that formula (4.4) holds. The same argument shows that (4.5) holds.

Part (3) follows from part (2) by a simple estimate. Indeed, definition, 
$x_{2}=f_{12}+2 f_{22}+f_{23}$ and $x_{3}=f_{13}+f_{23}+2 f_{33}$. Also $f_{22} f_{33}=f_{12} f_{33}=0$. Thus we see that $f_{12}+2 f_{22}+f_{23}+2 f_{33} \leq \max \left\{x_{2}, x_{3}\right\}$. This implies that inequality (4.6) follows from (4.4). Similarly, one shows that (4.7) follows from (4.5) and the fact that $x_{1} \geq f_{11}+f_{13}$. QED.
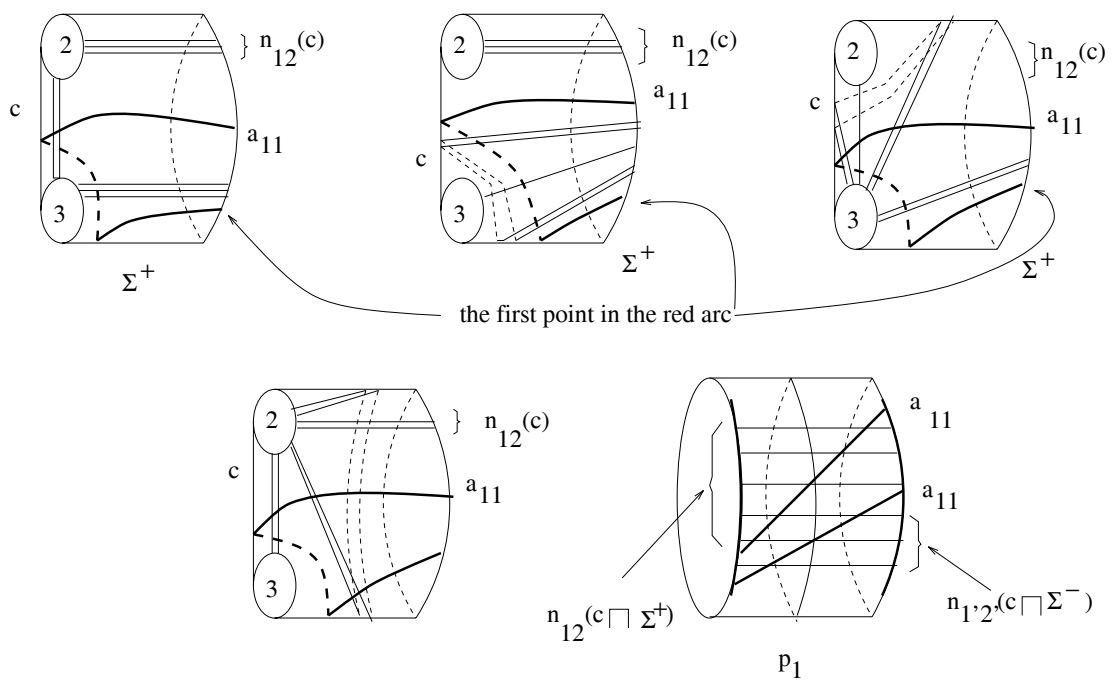

Figure 4.5

Now back to the proof of the proposition, if the twisting coordinate $t_{1}(\gamma) \geq 0$, we take $\delta=\left[a_{11} p_{1}^{e_{1}}\right]$ where $e_{1} \in\{0,1,2\}$ and consider $\gamma \delta$. By proposition 3.6, we can choose $e_{1}^{+}$and $e_{1}^{-} \in\{0,1\}$ so that the Dehn-Thurston coordinates of $\left(c\left(a_{11} p_{1}^{e_{1}^{ \pm}}\right) \cap \Sigma^{ \pm}\right)$in $C S^{g}\left(\Sigma^{ \pm}\right)$are of the form $\left(\left(x_{1}+2,0\right),\left(x_{i}, s_{i}\right),\left(x_{j}, s_{j}\right)\right)$ where $(i, j)$ are in $\left\{(2,3),\left(2^{\prime}, 3^{\prime}\right)\right\}$ and $\left|s_{i}\right|+\left|s_{j}\right| \leq 2$. Since their twisting coordinates at $p_{1}$ are zero, by taking $e_{1}=e_{1}^{+}+e_{1}^{-} \in\{0,1,2\}$, we conclude that the Dehn-Thurston coordinate of $c\left(a_{11} p_{1}^{e_{1}}\right)$ has the form $\left(\left[x_{1}+2,0\right],\left(x_{2}, s_{2}\right),\left(x_{3}, s_{3}\right),\left(x_{2^{\prime}}, s_{2^{\prime}}\right),\left(x_{3^{\prime}}, s_{3^{\prime}}\right)\right)$ where $\left|s_{2}\right|+\left|s_{3}\right| \leq 2$ and $\left|s_{2^{\prime}}\right|+\left|s_{3^{\prime}}\right| \leq 2$. (in fact proposition 3.4 shows that all $s_{i} \leq 0$.) By the above lemma, we have the associativity for the multiplication of the ordered three elements $p_{1}^{t_{1}}, \gamma_{z t}, \delta$. Thus $\gamma \delta=p_{1}^{t_{1}}\left(\gamma_{z t} \delta\right)$ has the Dehn-Thurston coordinate of the required form.

If the twisting coordinate $t_{1}(\gamma)<0$, we take $\delta=\left[p_{1}^{e_{1}} b_{11}\right]$ and consider $\gamma \delta$. The result follows by the same argument as above by proposition 3.6 and the above lemma.

It remains to show the inequality (4.1), we will consider $\delta=p_{1}^{s} b_{11}$ where $2 \geq s \geq 0$ for simplicity. The situation that $\delta=a_{11} p_{1}^{s}$ is similar and the proof will be omitted. 
First of all the intersection number

$$
\left.I\left(\alpha, p_{2}^{\left|s_{2}\right|} p_{3}^{\left|s_{3}\right|} p_{2^{\prime}}^{\left|s_{2^{\prime}}\right|} p_{3^{\prime}}^{\left|s_{3^{\prime}}\right|}\right) \leq 2 x_{2}(\alpha)+2 x_{3}(\alpha)+2 x_{2^{\prime}}(\alpha)+2 x_{3^{\prime}}(\alpha)\right)
$$

To estimate the first term $I(\alpha, \delta)$, by the triangle inequality (theorem $2.4(6))$ and inequality (4.7),

$$
\begin{aligned}
I(\alpha, \delta) & \leq I\left(\alpha, b_{11}\right)+|s| I\left(\alpha, p_{1}\right) \\
& \leq I\left(\alpha, b_{11}\right)+2 x_{1}(\alpha) \\
& \leq I\left(\alpha_{z t} p_{1}^{\left|t_{1}(\alpha)\right|}, b_{11}\right)+2 x_{1}(\alpha) \\
& \leq I\left(\alpha_{z t}, b_{11}\right)+I\left(p_{1}^{\left|t_{1}(\alpha)\right|}, b_{11}\right)+2 x_{1}(\alpha) \\
& \leq \max \left(x_{2}(\alpha), x_{3}(\alpha)\right)+\max \left(x_{2^{\prime}}(\alpha), x_{3^{\prime}}(\alpha)\right) \\
& \quad+2 x_{1}(\alpha)+2\left|t_{1}(\alpha)\right|+2 x_{1}(\alpha) \\
& \leq x_{2}(\alpha)+x_{3}(\alpha)+x_{2^{\prime}}(\alpha)+x_{3^{\prime}}(\alpha)+4 x_{1}(\alpha)+2\left|t_{1}(\alpha)\right| .
\end{aligned}
$$

Combining with (4.8), we see that (4.1) holds.

To see the second inequality $(4.2)$, we note that

$$
\begin{gathered}
\left(\left|s_{i}\right|+\left|s_{j}\right|\right) \max \left(I\left(\alpha, p_{i}\right), I\left(\alpha, p_{j}\right)\right)+\left(\left|s_{k}\right|+\left|s_{l}\right|\right) \max \left(I\left(\alpha, p_{k}\right), I\left(\alpha, p_{l}\right)\right) \\
\leq 4 \max \left(I\left(\alpha, p_{i}\right), I\left(\alpha, p_{j}\right)\right)+4 \max \left(I\left(\alpha, p_{k}\right), I\left(\alpha, p_{l}\right)\right)
\end{gathered}
$$

Thus, it remains to prove that

$$
\begin{aligned}
\left.I(\alpha, \delta) \leq 4 x_{1}(\alpha)+2\left|t_{1}(\alpha)\right|\right)+ & 2 \max \left(I\left(\alpha, p_{i}\right), I\left(\alpha, p_{j}\right)\right) \\
& +2 \max \left(I\left(\alpha, p_{k}\right), I\left(\alpha, p_{l}\right)\right) .
\end{aligned}
$$

Indeed, by the triangle inequality and inequality (4.7), we have

$$
\begin{aligned}
I(\alpha, \delta) & =I\left(\alpha, b_{11} p_{1}^{s}\right) \\
& \leq I\left(\alpha, b_{11}\right)+|s| I\left(\alpha, p_{1}\right) \\
& \leq I\left(\alpha, b_{11}\right)+2 x_{1}(\alpha) \\
\leq & I\left(p_{1}^{t_{1}(\alpha)} \alpha_{z t}, b_{11}\right)+2 x_{1}(\alpha) \\
\leq & I\left(p_{1}^{\left|t_{1}(\alpha)\right|}, b_{11}\right)+I\left(\alpha_{z t}, b_{11}\right)+2 x_{1}(\alpha) \\
\leq & \quad 2\left|t_{1}(\alpha)\right|+\max \left(x_{2}(\alpha), x_{3}(\alpha)\right)+\max \left(x_{2^{\prime}}(\alpha), x_{3^{\prime}}(\alpha)\right) \\
& \quad+2 x_{1}(\alpha)+2 x_{1}(\alpha) \\
& \leq 2 \max \left(x_{i}(\alpha), x_{j}(\alpha)\right)+2 \max \left(x_{k}(\alpha), x_{l}(\alpha)\right)+4 x_{1}(\alpha)+2\left|t_{1}(\alpha)\right|
\end{aligned}
$$

Combining with (4.9), we see that (4.2) holds. 
If $\{i, j\}=\{2,3\}$ and $\{k, l\}=\left\{2^{\prime}, 3^{\prime}\right\}$, then due to the inequalities that $\left|s_{2}\right|+\left|s_{3}\right| \leq 2$ and $\left|s_{2^{\prime}}\right|+\left|s_{3^{\prime}}\right| \leq 2$, we see that (4.9) becomes

$$
\begin{gathered}
\left(\left|s_{i}\right|+\left|s_{j}\right|\right) \max \left(I\left(\alpha, p_{i}\right), I\left(\alpha, p_{j}\right)\right)+\left(\left|s_{k}\right|+\left|s_{l}\right|\right) \max \left(I\left(\alpha, p_{k}\right), I\left(\alpha, p_{l}\right)\right) \\
\leq 2 \max \left(I\left(\alpha, p_{i}\right), I\left(\alpha, p_{j}\right)\right)+2 \max \left(I\left(\alpha, p_{k}\right), I\left(\alpha, p_{l}\right)\right)
\end{gathered}
$$

Combining (4.11) and (4.10), we see that inequality (4.3) holds.

Now the situation for $a_{11} p_{1}^{s}$ where $2 \geq s \geq 0$ is similar. We just replace all $b_{11}$ by $a_{11}$ in the above calculation. This ends the proof.

4.4. We now show that the $i$-th Dehn-Thurston coordinate $\left[x_{i}, t_{i}\right]$ is a continuous function of the intersection number functions. This is analogous to a non-trivial fact in the train-track approach to the space of measured laminations. Namely, given a good train-track $T$ and an edge $E$ in $T$, there exists a finite collection of simple loops $s_{1}, \ldots, s_{m}$ and a continuous homogeneous function $f$ so that for all curve systems $\alpha$ carried by $T$, the weight of $\alpha$ at the edge $E$ is $f\left(I\left(\alpha, s_{1}\right), \ldots, I\left(\alpha, s_{m}\right)\right)$.

We shall use the same the notations as in $\S 4.2$ and $\S 4.3$.

Proposition. (1) There exists a continuous homogeneous function $f\left(x_{1}, x_{2}, x_{3}, x_{4}\right) \quad: \quad \mathbf{R}^{4} \rightarrow R^{2} / \pm$ so that for any $\alpha$ in $C S^{g}\left(\Sigma_{1,1}\right)$, the first Dehn-Thurston coordinate of $\alpha$ is $\left[x_{1}(\alpha), t_{1}(\alpha)\right]=$ $f\left(x_{1}(\alpha), x_{2}(\alpha), I\left(\alpha, p_{1} b_{2}\right), I\left(\alpha, b_{2} p_{1}\right)\right)$.

(2) There exists a continuous homogeneous function $f\left(x_{1}, x_{2}, x_{3}, \ldots x_{7}\right)$ : $\mathbf{R}^{7} \rightarrow R^{2} / \pm$ so that for any $\alpha$ in $C S^{g}\left(\Sigma_{0,4}\right)$, the first Dehn-Thurston coordinate of $\alpha$ is $\left[x_{1}(\alpha), t_{1}(\alpha)\right]=f\left(x_{1}(\alpha), x_{2}(\alpha), x_{3}(\alpha), x_{2^{\prime}}(\alpha), x_{3^{\prime}}(\alpha), I\left(\alpha, a_{11}\right)\right.$, $\left.I\left(\alpha, b_{11}\right)\right)$.

Corollary. The Dehn-Thurston coordinate on $C S(\Sigma)$ can be extended to be a continuous homogeneous function from $M L(\Sigma)$ to $\left(\mathbf{R}^{2} / \pm\right)^{3 g+r-3} \times \mathbf{R}_{\geq 0}^{r}$.

Indeed, by the proposition, it suffices to show that for each $\alpha$ in $C S(\Sigma)$, the intersection number function $I(\alpha,):. C S(\Sigma) \rightarrow \mathbf{R}$ can be extended to be a continuous homogeneous function on $M L(\Sigma)$. But this follows from the definition of the topology of $M L(\Sigma)$.

Proof of the proposition. We will prove part (2) in detail. The same argument also applies to part (1).

Write $\alpha=p_{1}^{t_{1}} \alpha_{z t}$ where $t_{1}=t_{1}(\alpha)$ and $\alpha_{z t}$ is the standard twistingzero representative of $\alpha$. Let $a_{11}$ and $b_{11}$ be the same simple loops used in $\S 4.3$ (with Dehn-Thurston coordinates $([2,0],(0,0),(0,0),(0,0),(0,0))$ and $([2,-2],(0,0),(0,0),(0,0),(0,0))$. By lemma $4.3(1)$, we know that both $\left(p_{1}^{n}, \alpha_{z t}, a_{11}\right)$ and $\left(b_{11}, \alpha_{z t}, p_{1}^{n}\right)$ contain neither positive triangles nor positive 
quadrilaterals for $n \geq 1$. Thus by theorem $2.4(7)$, if $t_{1} \geq 0$,

$$
I\left(\alpha, a_{11}\right)=I\left(p_{1}^{t_{1}} \alpha_{z t}, a_{11}\right)=I\left(p_{1}^{t_{1}}, a_{11}\right)+I\left(\alpha_{z t}, a_{11}\right)=2 t_{1}+I\left(\alpha_{z t}, a_{11}\right) .
$$

Also if $t_{1} \leq 0$, then $I\left(\alpha, b_{11}\right)=I\left(p_{1}^{t_{1}} \alpha_{z t}, b_{11}\right)=I\left(p_{1}^{t_{1}}, b_{11}\right)+I\left(\alpha_{z t}, b_{11}\right)=$ $-2 t_{1}+I\left(\alpha_{z t}, b_{11}\right)$. This shows that

$$
t_{1}(\alpha)= \begin{cases}\frac{1}{2} I\left(\alpha, a_{11}\right)-\frac{1}{2} I\left(\alpha_{z t}, a_{11}\right), & \text { if } t_{1}(\alpha) \geq 0 \\ -\frac{1}{2} I\left(\alpha, b_{11}\right)+\frac{1}{2} I\left(\alpha_{z t}, b_{11}\right), & \text { if } t_{1}(\alpha) \leq 0\end{cases}
$$

Now by lemma 4.3(2), we can express explicitly that the intersection numbers $I\left(\alpha_{z t}, x\right)$ for $x=a_{11}$ or $b_{11}$ in terms of continuous homogeneous functions in the coordinates $x_{i}\left(i \in\left\{1,2,3,2^{\prime}, 3^{\prime}\right\}\right), I\left(\alpha, a_{11}\right)$, and $I\left(\alpha, b_{11}\right)$. This shows that $\left[x_{1}(\alpha), t_{1}(\alpha)\right]$ can be expressed as a piecewise linear homogeneous function in seven variables $x_{1}, x_{2}, x_{3}, x_{2^{\prime}}, x_{3^{\prime}}, I\left(\alpha, a_{11}\right)$ and $I\left(\alpha, b_{11}\right)$. One checks easily that the function is continuous. Thus the result follows.

\section{Proof of Theorem 1.1.}

Recall that $\Sigma=\Sigma_{g, r}$ is a compact oriented surface with a colored $\mathrm{H}$ decomposition and $\Pi: C S(\Sigma) \rightarrow\left(\mathbf{Z}^{2} / \pm\right)^{3 g+r-3} \times \mathbf{Z}_{\geq 0}$ is the associated Dehn-Thurston coordinate. Our goal is to prove that

$$
|I(\alpha, \beta)-I(\alpha, \gamma)| \leq K_{2,3}|\Pi(\alpha)||\Pi(\beta)-\Pi(\gamma)|
$$

for all $\alpha, \beta$ and $\gamma$ in $C S(\Sigma)$ where $K_{2,3}=2$ if the 3-holed sphere decomposition contains only embedded 4 -holed spheres and $K_{2,3}=3$ in the rest of the cases.

First we note that inequality (5.1) is homogeneous in $\alpha, \beta$ and $\gamma$. Thus it suffices to show

$$
\left|I\left(\alpha^{2}, \beta^{2}\right)-I\left(\alpha^{2}, \gamma^{2}\right)\right| \leq K_{2,3}\left|\Pi\left(\alpha^{2}\right)\right|\left|\Pi\left(\beta^{2}\right)-\Pi\left(\gamma^{2}\right)\right|
$$

We call an element $\alpha$ in $C S(\Sigma)$ even if its Dehn-Thurston coordinate $\Pi(\alpha)=\left(\left[x_{1}, t_{1}\right], \ldots,\left[x_{3 g+r-3}\right.\right.$,

$\left.\left.t_{3 g+r-3}\right], x_{3 g+r-2}, \ldots, x_{3 g+2 r-3}\right)$ has the property that all $x_{i}$ and $t_{j}$ are even integers. Denote the set of all even classes in $C S(\Sigma)$ by $C S_{\text {even }}$ and let $Z_{\text {even }}$ be $\left\{\left(\left[x_{1}, t_{1}\right], \ldots,\left[x_{3 g+r-3}, t_{3 g+r-3}\right], x_{3 g+r-2}, \ldots, x_{3 g+2 r-3}\right) \in\left(\mathbf{Z}^{2} / \pm\right)^{3 g+r-3} \times \mathbf{Z}_{\geq 0}^{r} \mid\right.$ all $x_{i}$ and $t_{j}$ are even\}. Thus by proposition 2.5, the Dehn-Thurston coordinate $\Pi$ is a bijection from $C S_{\text {even }}$ to $Z_{\text {even }}$. Since $\alpha^{2}$ is always in $C S_{\text {even }}$, thus it suffices to prove the inequality (5.1) for all even classes $\alpha, \beta$ and $\gamma$. This will be the goal of the proof in the rest of the section. 
5.1. We will use the following simple lemma.

Lemma. If $u, v$ are in $Z_{\text {even }}$ so that $|u-v|=2 n$, then there exist $n+1$ points $u_{0}=u, u_{1}, \ldots, u_{n}=v$ in $Z_{\text {even }}$ so that $\left|u_{i}-u_{i+1}\right|=2$.

The proof of it is evident from the definition of the metric.

5.2. As a consequence, we have,

Lemma. If the inequality (5.1) holds for all $\beta, \gamma \in C S_{\text {even }}$ so that $\mid \Pi(\beta)-$ $\Pi(\gamma) \mid=2$ then it holds for all classes $\alpha, \beta$ and $\gamma$ in $C S_{\text {even }}$.

Indeed, by proposition 2.5 and lemma 5.1, for any two classes $\beta, \gamma \in$ $C S_{\text {even }}$ so that $|\Pi(\beta)-\Pi(\gamma)|=2 n$, there exists a sequence of $n+1$ even classes $\beta_{0}=\beta, \beta_{1}, \ldots, \beta_{n}=\gamma$ so that $\left|\Pi\left(\beta_{i}\right)-\Pi\left(\beta_{i+1}\right)\right|=2$. Thus $\mid I(\alpha, \beta)-$ $I(\alpha, \gamma)\left|\leq \sum_{i=1}^{n}\right| I\left(\alpha, \beta_{i}\right)-I\left(\alpha, \beta_{i-1}\right)\left|\leq K_{2,3} \sum_{i=1}^{n}\right| \Pi(\alpha)|| \Pi\left(\beta_{i}\right)-\Pi\left(\beta_{i-1}\right) \mid \leq$ $2 K_{2,3} n|\Pi(\alpha)|=K_{2,3}|\Pi(\alpha)||\Pi(\beta)-\Pi(\gamma)|$.

5.3. It remains to prove the inequality (5.1) for even classes $\beta$ and $\gamma$ in $C S_{\text {even }}(\Sigma)$ so that $|\Pi(\beta)-\Pi(\gamma)|=2$. Since they are both even vectors in $Z_{\text {even }}$, their coordinates are the same except in one position which differs by 2. Thus either there is one $i$ so that $x_{i}(\beta)=x_{i}(\gamma) \pm 2$ or there is one index $j$ so that $t_{j}(\beta)=t_{j}(\gamma) \pm 2$ and all other coordinates are the same.

In the case $t_{j}(\beta)=t_{j}(\gamma) \pm 2$, say $t_{j}(\beta)=t_{j}(\gamma)+2$, then by the definition of twisting coordinate, we have $\beta=\left[p_{j}^{2}\right] \gamma$. Thus by the triangle inequality (theorem 2.4(6))

$$
\begin{aligned}
|I(\alpha, \beta)-I(\alpha, \gamma)| & =\left|I\left(\alpha,\left[p_{2}^{2}\right] \gamma\right)-I(\alpha, \gamma)\right| \leq I\left(\alpha, p_{j}^{2}\right) \\
& =2 x_{j}(\alpha) \leq 4|\Pi(\alpha)| \leq 2 K_{2,3}|\Pi(\alpha)|
\end{aligned}
$$

In the case that $\left|x_{i}(\beta)-x_{i}(\gamma)\right|=2$, we need to discuss two subcases that $i \leq 3 g+r-3$ or $i \geq 3 g+r-2$.

5.4. If $\left|x_{i}(\beta)-x_{i}(\gamma)\right|=2$ so that $i \leq 3 g+r-3$, say for simplicity that $i=1$ and $x_{1}(\beta)=x_{1}(\gamma)+2$, let $\Sigma^{\prime}$ be the 1-holed torus or the 4-holed sphere which is the component of $\Sigma-\cup_{j \neq 1} i n t\left(N\left(p_{j}\right)\right)$. Without loss of generality, let the boundary components of $\Sigma^{\prime}$ be parallel to $p_{i_{2}}, \ldots, p_{i_{s}}$ where $s=2$ or 5 and some $i_{j}$ may be $i_{k}$ but no three of these indices are the same. See figure 5.1 . 


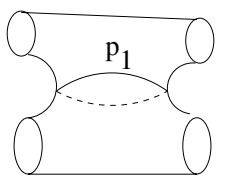

four distinct boundary components

(a)

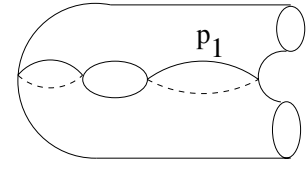

(b)

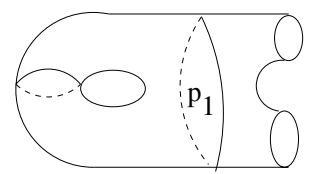

(d)

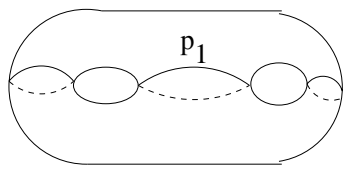

(c)

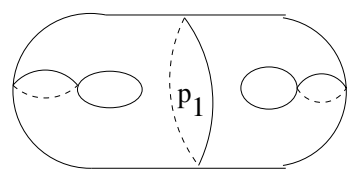

(e)

non-embedded 4-holed spheres in the 3-holed sphere decomposition

Figure 5.1

Take representatives $b \in \beta$ and $c \in \gamma$ so that $b \cap \Sigma^{\prime}$ and $c \cap \Sigma^{\prime}$ are good curve systems in $\Sigma^{\prime}$ and the twisting coordinates of $b \cap \Sigma^{\prime}$ and $c \cap \Sigma^{\prime}$ at the boundary components of $\Sigma^{\prime}$ are zero (this can always be achieved by an isotopy of $b$ and $c$ in $\Sigma$.) Now by the construction, the Dehn-Thurston coordinates of $\left[b \cap \Sigma^{\prime}\right]$ and $\left[c \cap \Sigma^{\prime}\right]$ in $C S^{g}\left(\Sigma^{\prime}\right)$ differ only in the $x_{1}$-coordinate by 2 . Thus by propositions 4.2 and 4.3 , we find $\delta \in C S_{0}\left(\Sigma^{\prime}\right)$ so that

$$
\beta=\prod_{j=2}\left[p_{i_{j}}^{e_{j}}\right]\left(\delta^{ \pm 1} \gamma\right)
$$

where $e_{j} \in\{0, \pm 1, \pm 2\}$ (note that if $\Sigma^{\prime} \cong \Sigma_{1,1}$, we apply proposition 4.2 twice to get this form.) Furthermore for all $\alpha$ in $C S(\Sigma)$, by the triangle inequality (theorem 2.4(6)) we obtain that

$$
\begin{aligned}
|I(\alpha, \beta)-I(\alpha, \gamma)| & =\left|I\left(\alpha, \prod_{j=2}^{s}\left[p_{i_{j}}^{e_{j}}\right]\left(\delta^{ \pm} \gamma\right)\right)-I(\alpha, \gamma)\right| \\
& \leq I(\alpha, \delta)+I\left(\alpha, \prod_{j=2}^{s}\left[p_{i_{j}}^{\left|e_{j}\right|}\right]\right) .
\end{aligned}
$$


We claim that

$$
I(\alpha, \delta)+I\left(\alpha, \prod_{j=2}^{s}\left[p_{i_{j}}^{\left|e_{j}\right|}\right]\right) \leq 2 K_{2,3}|\Pi(\alpha)| .
$$

To prove this, we need to discuss several cases.

Case (1), the boundary components of $\Sigma^{\prime}$ are pairwise non-isotopic in the surface $\Sigma$, i.e., $\left\{p_{i_{1}}, \ldots, p_{i_{s}}\right\}$ are pairwise distinct. Then by propositions 4.2 and inequality (4.1),

$$
I(\alpha, \delta)+I\left(\alpha, \prod_{j=2}^{s}\left[p_{i_{j}}^{\left|e_{j}\right|}\right]\right) \leq 4|\Pi(\alpha)| .
$$

Case (2), the surface $\Sigma^{\prime}$ is the 4 -holed sphere and $\left\{p_{i_{2}}, \ldots, p_{i_{5}}\right\}$ consists of two elements. Let $\partial \Sigma^{\prime}=\partial_{2}, \partial_{3}, \partial_{2^{\prime}}, \partial_{3^{\prime}}$. Then $\left\{2,3,2^{\prime}, 3^{\prime}\right\}$ is partitioned into a 2-element set $\{i, j\} \cup\{k, l\}$ according to the isotopy classes of $\partial \Sigma^{\prime}$ in $\Sigma$. In particular, $x_{i}(\alpha)=x_{j}(\alpha)$ and $x_{k}(\alpha)=x_{l}(\alpha)$. Let $s_{r}$ be the index $e_{t}$ associated to the boundary component $\partial_{r}=p_{i_{t}}$. Let $x_{r}=x_{r}(\alpha)$. Then we have,

$$
\begin{aligned}
I(\alpha, \delta)+I\left(\alpha, \prod_{j=2}^{5}\left[p_{i_{j}}^{\left|e_{j}\right|}\right]\right) & =I(\alpha, \delta)+I\left(\alpha, \prod_{r=2}^{5}\left[\partial_{r}^{s_{r}}\right]\right) \\
& =I(\alpha, \delta)+\left(\left|s_{i}\right|+\left|s_{j}\right|\right) x_{i}+\left(\left|s_{k}\right|+\left|s_{l}\right|\right) x_{k} \\
& \leq I(\alpha, \delta)+\left(\left|s_{i}\right|+\left|s_{j}\right|\right) \max \left(x_{i}, x_{j}\right) \\
& \quad+\left(\left|s_{k}\right|+\left|s_{l}\right|\right) \max \left(x_{k}, x_{l}\right) \\
& \leq 4 x_{1}+2\left|t_{1}(\alpha)\right|+6 \max \left(x_{i}, x_{j}\right)+6 \max \left(x_{k}, x_{l}\right) \\
& \leq 4 x_{1}+2\left|t_{1}(\alpha)\right|+6 x_{i}+6 x_{k} \\
& \leq 6|\Pi(\alpha)| .
\end{aligned}
$$

If $\{i, j\}=\{2,3\}$ and $\{k, l\}=\left\{2^{\prime}, 3^{\prime}\right\}$, then we use inequality (4.3) and obtain $I(\alpha, \delta)+I\left(\alpha, \prod_{j=2}^{5}\left[p_{i_{j}}^{e_{j}}\right]\right) \leq 4|\Pi(\alpha)|$.

This shows that (5.3) holds.

Case (3), the surface $\Sigma^{\prime}$ is a 4 -holed sphere and the set $\left\{p_{i_{2}}, \ldots, p_{i_{5}}\right\}$ 
consists of three elements. Say $\left[\partial_{i}\right]=\left[\partial_{j}\right]$. In particular $x_{i}=x_{j}$. Then

$$
\begin{aligned}
I(\alpha, \delta)+I\left(\alpha, \prod_{j=2}^{5}\left[p_{i_{j}}^{\left|e_{j}\right|}\right]\right) & =I(\alpha, \delta)+I\left(\alpha, \prod_{r}^{5}\left[\partial_{r}^{\left|s_{r}\right|}\right]\right) \\
& \leq I(\alpha, \delta)+\left(\left|s_{i}\right|+\left|s_{j}\right|\right) x_{i}+\left|s_{k}\right| x_{k}+\left|s_{l}\right| x_{l} \\
& \leq I(\alpha, \delta)+\left(\left|s_{i}\right|+\left|s_{j}\right|\right) \max \left(x_{i}, x_{j}\right) \\
& \quad+\left(\left|s_{k}\right|+\left|s_{l}\right|\right) \max \left(x_{k}, x_{l}\right) \\
& \leq 4 x_{1}+2\left|t_{1}(\alpha)\right|+6 \max \left(x_{i}, x_{j}\right)+6 \max \left(x_{k}, x_{l}\right) \\
& \leq 4 x_{1}+2\left|t_{1}(\alpha)\right|+6\left(x_{i}+x_{k}+x_{l}\right) \\
& \leq 6|\Pi(\alpha)| .
\end{aligned}
$$

If $\{i, j\}=\{2,3\}$ and $\{k, l\}=\left\{2^{\prime}, 3^{\prime}\right\}$, then we use inequality (4.3) and obtain $I(\alpha, \delta)+I\left(\alpha, \prod_{j=2}^{5}\left[p_{i_{j}}^{\left|e_{j}\right|}\right]\right) \leq 4|\Pi(\alpha)|$.

Thus (5.3) follows.

5.5. If $x_{i}(\beta)=x_{i}(\gamma)+2$ for some $i \geq 3 g+r-2$, then $p_{i}$ is a boundary component of the surface $\Sigma$. In this case, by simply doubling the surface across the boundary, we reduce the situation to cases in $\S 5.3$ and $\S 5.4$.

To be more precise, let $\Sigma^{*}$ be the closed surface obtained by doubling the surface $\Sigma$ across its boundary. We give $\Sigma^{*}$ the colored hexagonal decompositioin by doubling the one from $\Sigma$. If $s$ is a curve system on $\Sigma$, let $s^{*}$ be its double in $\Sigma^{*}$ which has zero twisting coordinates at all components of $\partial \Sigma$. Define $[a]^{*}$ to be $\left[a^{*}\right]$. Clearly we have $\left|\Pi\left(s^{*}\right)\right| \leq 2|\Pi(s)|$ and $2 I(a, b)=I\left(a^{*}, b^{*}\right)$. Furthermore, by the construction, we have $\left|\Pi\left(\beta^{*}\right)-\Pi\left(\gamma^{*}\right)\right|=2$. Thus $|I(\alpha, \beta)-I(\alpha, \gamma)|=1 / 2\left|I\left(\alpha^{*}, \beta^{*}\right)-I\left(\alpha^{*}, \gamma^{*}\right)\right| \leq$ $1 / 2 K_{2,3}\left|\Pi\left(\alpha^{*}\right)\right|\left|\Pi\left(\beta^{*}\right)-\Pi\left(\gamma^{*}\right)\right| \leq K_{2,3}|\Pi(\alpha)||\Pi(\beta)-\Pi(\gamma)|$. This ends the proof.

\section{The Space of Measured Laminations on Surfaces.}

The goal of this section is to reestablish the basic results in the theory of measured laminations on surfaces from the Cauchy inequality. A crucial fact used in the proof is the corollary 4.4 that the $i$-th Dehn-Thurston coordinate $\left[x_{i}, t_{i}\right]$ is a continuous homogeneous function defined on the space $M L(\Sigma)$.

6.1. Given a compact oriented surface $\Sigma=\Sigma_{g, r}$ of negative Euler number, we fix a colored H-decomposition of it. We shall identify the space $C S(\Sigma)$ with its image under Thurston's embedding $\Theta(C S(\Sigma))$, and define $I$ on the image $\Theta(C S(\Sigma))$ by $I(x, y)=I\left(\Theta^{-1}(x), \Theta^{-1}(y)\right)$. Extend the intersection pairing $I$ to $\left(\mathbf{Q}_{\geq 0} \times \Theta(C S(\Sigma))\right)^{2} \rightarrow \mathbf{Q}$ by homogeneity. Let 
$\Phi:\left(2 \mathbf{Z}^{2} / \pm\right)^{3 g+r-3} \times\left(2 \mathbf{Z}_{\geq 0}\right)^{r} \rightarrow \Theta(C S(\Sigma))$ be the composition $\Theta\left(\Pi^{-1} \mid\right)$. Due to the homogeneity, we can extend $\Phi$ to a homogeneous map, still denoted by $\Phi$, defined from $\left(\mathbf{Q}^{2} / \pm\right)^{3 g+r-3} \times \mathbf{Q}_{\geq 0}^{r}$ to $\mathbf{Q}_{\geq 0} \times \Theta(C S(\Sigma))$. Theorem 1.1 extends to the classes in $\left(\mathbf{Q}^{2} / \pm\right)^{3 g+r-3} \times \mathbf{Q}_{\geq 0}^{r}$ and we have

$$
|I(\Phi(x), \Phi(y))-I(\Phi(x), \Phi(z))| \leq K_{2,3}|x||y-z|
$$

for all $x, y, z$ in $\left(\mathbf{Q}^{2} / \pm\right)^{3 g+r-3} \times \mathbf{Q}_{>0}^{r}$.

Note that the metric completion of $\left(\mathbf{Q}^{2} / \pm\right)^{3 g+r-3} \times \mathbf{Q}_{\geq 0}^{r}$ under the metric $|x-y|$ is $\left(\mathbf{R}^{2} / \pm\right)^{3 g+r-3} \times \mathbf{R}_{\geq 0}^{r}$. Also the product topology on $\mathbf{R}_{\geq 0}^{C S(\Sigma)}$ is metrizable and a metric can be chosen to be complete. In the following discussion, we shall fix a complete metric on the space $\mathbf{R}_{\geq 0}^{C S(\Sigma)}$ and give $M L(\Sigma)$ the induced complete metric.

6.2. One of the basic point-set topological fact we will use is the following lemma. The proof can be found in most books on metric space and will be omitted.

Lemma. Suppose $X$ and $Y$ are complete metric spaces and $Z \subset X$ is a dense subspace. If $f: Z \rightarrow Y$ is a map sending all Cauchy sequences in $Z$ to Cauchy sequences in $Y$, then $f$ can be extended to be a continuous function from $X$ to $Y$.

6.3. We now prove the first basic result in the theory of measured laminations.

Proposition. The map $\Phi$ extends to a homeomorphism from the space $\left(\mathbf{R}^{2} / \pm\right)^{3 g+r-3} \times \mathbf{R}_{\geq 0}^{r}$ to $M L(\Sigma)$.

Proof. To show that $\Phi$ from $\left(\mathbf{Q}^{2} / \pm\right)^{3 g+r-3} \times \mathbf{Q}_{\geq 0}^{r}$ to $M L(\Sigma)$ can be extended to $\left(\mathbf{R}^{2} / \pm\right)^{3 g+r-3} \times \mathbf{R}_{\geq 0}^{r} \rightarrow M L(\Sigma)$, take any $\alpha$ in $C S(\Sigma)$, and a Cauchy sequence $\left\{x_{n}\right\}$ in $\left(\mathbf{Q}^{2} / \pm\right)^{3 g+r-3} \times \mathbf{Q}_{\geq 0}^{r}$. Then by the inequality (6.1) for $x=\Phi^{-1}(\alpha), y=x_{n}, z=x_{m}$, we see that $I\left(\Phi\left(x_{n}\right), \alpha\right)$ is again a Cauchy sequence in $\mathbf{R}$. Thus it converges for all $\alpha$. Thus, by the definition of the product topology on $M L(\Sigma)$, we see that $\phi\left(x_{n}\right)$ is convergent in $M L(\Sigma)$. By lemma 6.2 , this shows that $\Phi$ can be extended to be a continuous function from $\left(\mathbf{R}^{2} / \pm\right)^{3 g+r-3} \times \mathbf{R}_{\geq 0}^{r}$ to $M L(\Sigma)$.

Now by the continuity of the i-th Dehn-Thurston coordinate $\left[x_{i}, t_{i}\right]$ (corollaries 4.4), we see that the $i$-th coordinate can be extended to be a homogeneous continuous function defined on $M L(\Sigma)$. Thus we obtain a continuous function $\Psi: M L(\Sigma) \rightarrow\left(\mathbf{R}^{2} / \pm\right)^{3 g+r-3} \times \mathbf{R}_{>0}^{r}$ which is the continuous extension of the Dehn-Thurston coordinate on $\mathbf{Q} \times \Theta(C S(\Sigma))$. By definition, on the dense subspaces $\left(\mathbf{Q}^{2} / \pm\right)^{3 g+r-3} \times \mathbf{Q}_{\geq 0}^{r}$ and $\mathbf{Q}_{\geq 0} \times \Theta(C S(\Sigma))$, we have 
$\Phi \Psi=i d$ and $\Psi \Phi=i d$. Thus by the continuity, $\Phi \Psi=i d$ and $\Psi \Phi=i d$ on the whole spaces $\left(\mathbf{R}^{2} / \pm\right)^{3 g+r-3} \times \mathbf{R}_{\geq 0}^{r}$ and $M L(\Sigma)$. This establishes the assertion that Dehn-Thurston coordinate $\Pi$ is a homeomorphism from $M L(\Sigma)$ to $\left(\mathbf{R}^{2} / \pm\right)^{3 g+r-3} \times \mathbf{R}_{>0}^{r}$. Furthermore, the space of compactly supported measured laminations $M L_{0}(\Sigma)$ which is the closure of $\mathbf{Q}_{\geq 0} \times \Theta\left(C S_{0}(\Sigma)\right)$ is homeomorphic to $\mathbf{R}^{6 g+2 n-6} \times(0, . ., 0)$ under the Dehn-Thurston coordinate.

6.4. Finally, we show that the intersection pairing $I$ extends to a continuous homogeneous map from $M L(\Sigma) \times M L(\Sigma)$ to $\mathbf{R}$. By lemma 6.2, it suffices to show that for any Cauchy sequences $\left\{x_{n}\right\}$ and $\left\{y_{n}\right\}$ in $\mathbf{Q}_{\geq 0} \times \Theta(C S(\Sigma))$, the sequence $I\left(x_{n}, y_{n}\right)$ is convergent. Indeed, by the continuity of the DehnThurston map $\Pi$, both sequences $\Psi\left(x_{n}\right)$ and $\Psi\left(y_{n}\right)$ are convergent and in particular bounded, say by $M$. Thus, we have

$$
\begin{gathered}
\left|I\left(x_{n}, y_{n}\right)-I\left(x_{m}, y_{m}\right)\right| \leq\left|I\left(x_{n}, y_{n}\right)-I\left(x_{n}, y_{m}\right)\right|+\left|I\left(x_{n}, y_{m}\right)-I\left(x_{m}, y_{m}\right)\right| \\
\leq 3\left|\Psi\left(x_{n}\right)\right|\left|\Psi\left(y_{n}\right)-\Psi\left(y_{m}\right)\right|+3\left|\Psi\left(y_{m}\right)\right|\left|\Psi\left(x_{n}\right)-\Psi\left(x_{m}\right)\right| \\
\leq 3 M\left(\left|\Psi\left(y_{n}\right)-\Psi\left(y_{m}\right)\right|+\left|\Psi\left(x_{n}\right)-\Psi\left(x_{m}\right)\right|\right) .
\end{gathered}
$$

This shows that $I\left(x_{n}, y_{n}\right)$ is still a Cauchy sequence. Thus by lemma 6.2 , the pairing $I$ extends continuously to the product space $M L(\Sigma) \times M L(\Sigma)$. We remark that by continuity, the inequality (6.1) still holds for all $x, y, z \in$ $M L(\Sigma)$.

\section{Appendix: A Proof of Theorem 2.4.}

We reproduce a proof of the basic property of the multiplication of curve systems in this appendix for completeness. We begin with the following lemma which shows that the multiplication is well defined.

Lemma. (a) If $a$ and $b$ are curve systems with $|a \cap b|=I(a, b)$, then the submanifold $a b$ obtained by resolving all intersection points between $a$ and $b$ from $a$ to $b$ is a curve system.

(b) Suppose $a, b$ and $c$ are curve systems in $\Sigma$ so that $|a \cap b|=I(a, b)$, $|b \cap c|=I(b, c),|c \cap a|=I(c, a)$ and $|a \cap b \cap c|=0$. If there are no positive triangles or positive quadrilaterals in the ordered triple $(a, b, c)$, then $|a b \cap c|=I(a b, c) \mid$ and $|a \cap b c|=I(a, b c) \mid$.

Proof. (a) If $a b$ is not a curve system, then there exists either (1) a simple closed curve $s$ in $a b$ and an annulus $D$ with $\partial D=s \cup d$ where $d$ is a boundary component of $\Sigma$ or (2) a simple closed curve $s$ in $a b$ bounding a disc $D$ in $\Sigma$, or (3) there exists an arc component $s$ in $a b$ and a disk $D$ in the surface 
so that $\partial D=s \cup d$ where $s \cap d=\partial s=\partial d$ and $d$ is an arc in $\partial \Sigma$. By replacing $s$ and finding another component of $a b$ in int $(D)$ if necessary, we may assume that $a b \cap i n t(D)=\emptyset$. Take a small regular neighborhood $N(a \cup b)$ of $a \cup b$ to be $N(a) \cup N(b)$. We assume the resolutions are taken place inside $N(a) \cap N(b)$. Thus int $(D)$ contains a finite number of connected components $R_{0}, R_{1}, \ldots, R_{n}$ of $\Sigma-\operatorname{int}(N(a) \cup N(b))$. These regions are so labeled that they satisfy the conditions below, (i) if $i \geq 1$ then $R_{i} \neq \emptyset$ and $R_{i} \cap \partial \Sigma=\emptyset$; and (ii) $R_{0}=\emptyset$ in the case (2), and in the cases (1) and (3), $R_{0}$ is the region which intersects $\partial \Sigma$; (iii) the region $R_{0}$ is a disc if $D$ is a disc intersecting $\partial \Sigma$ and $R_{0}$ is an annulus if $D$ is an annulus. Each region $R_{i}(i \geq 1)$ is a disc since otherwise there would be at least two boundary components of $R_{i}$ in $\operatorname{int}(D)$. This would contradict the assumption that $\operatorname{int}(D) \cap a b=\emptyset$. Call a point in $\partial N(a) \cap \partial N(b)$ a corner of $N(a \cup b)$. Each point $p$ in $a \cap b$ corresponds to four corners in $\partial N(p)$ where $N(p)$ is the connected component of $N(a) \cap N(b)$ containing $p$. These four corners form two pairs of opposite corners in the quadrilateral $\partial N(p)$. Join opposite corners in $\partial N(p)$ by an arc in $\operatorname{int}(N(p))$ so that it avoids one of the resolutions of $a \cap b$ at $p$. We call the arc a bridge between the corners. A corner of $\partial N(a \cup b)$ in a region $R_{i}$ is called a vertex of $R_{i}$. Vertices of $R_{i}$ decompose $\partial R_{i}$ into edges. Each edge is either in $\partial N(a)$, or in $\partial N(b)$, or in $\partial \Sigma$. There is at most one edge in $R_{0}$ which is in $\partial \Sigma$ in the cases (1) and (3). If two edges have a vertex in common, they cannot be both in $N(a)$ (resp. in $N(b)$ ). Thus for $i \geq 1$, there are even number of edges in $R_{i}$. Each region $R_{i}$ with $i \geq 1$, must have at least four edges since $|a \cap b|=I(a, b)$ (if there were regions with only two edges, then the region provides a Whitney disc for $a \cup b$ ). More importantly, the definition of the resolution implies the following alternating principle: if $v$ and $v^{\prime}$ are two vertices joint by an edge in $R_{i}(i \geq 0)$ so that the edge is either in $N(a)$ or in $N(b)$ then exactly one of the bridges from $v$ or $v^{\prime}$ still lies in $D$ (see figure $\mathrm{A} 1(\mathrm{~b}))$.

Form a graph $G$ in $D$ by putting a 0 -cell in each $\operatorname{int}\left(R_{i}\right), i \geq 0$. Joint two 0 -cells of $\operatorname{int}\left(R_{i}\right)$ and $\operatorname{int}\left(R_{j}\right)$ by a 1-cell in $D$ if there are opposite vertices in $R_{i}$ and $R_{j}$ so that their bridge is in $D$ (the 1-cell is an extension of the bridge). These 1-cells are chosen to be pairwise disjoint except at the end points. By the construction, if $D$ is a disc, the graph $G$ is homotopic to $D$ since each region $R_{i}$ is a disc; if $D$ is an annulus, the region $R_{0}$ is an annulus, thus the graph $G$ is again homotopic to a disc. In both cases, $G$ is a tree. Therefore either $G$ is a point or $G$ contains two 0-cells of valency one. However by the construction, each region $R_{i}(i \geq 1)$ has at least four edges and thus corresponds to a 0 -cell of valency at least two by the alternating principle. 


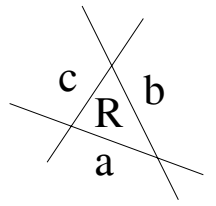

(a1)

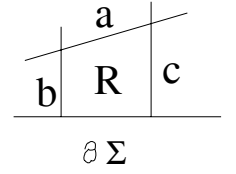

(a2)

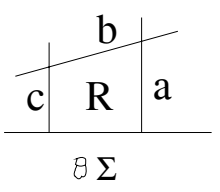

(a3)

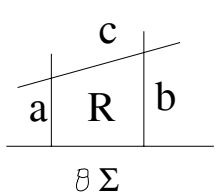

(a4)

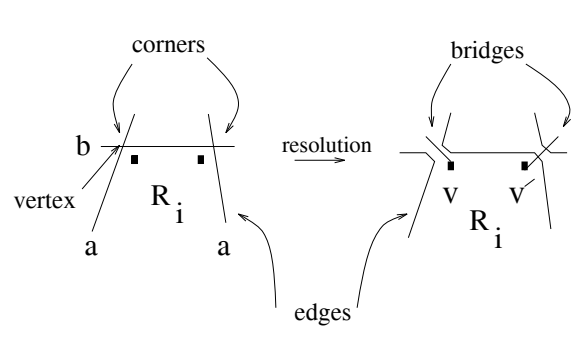

(b)

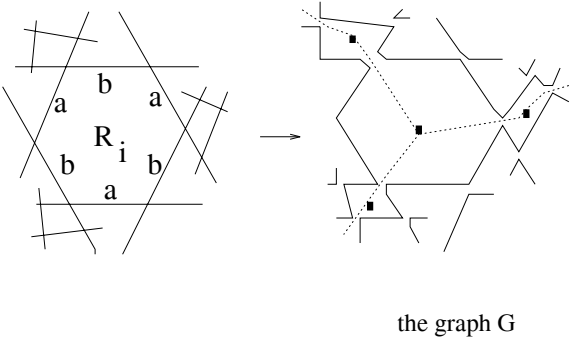

(c)

Figure A1

Thus the graph $G$ must be a point. Therefore, there is only one region $R_{0}$ which has at most one vertex by the alternating principle. This contradicts the condition that $|a \cap b|=I(a, b)$. (b) Suppose the result is false, say that $|a b \cap c|>I(a b, c)$. Then there is a disc $D \subset \Sigma$ so that either (1) $\partial D$ is a union of two arcs $s$ and $t$ with $s \cap t=\partial s=\partial t, s \subset c$ and $t \subset a b$, or (2) $\partial D$ is a union of three $\operatorname{arcs} s, t, u$ so that each pair of arcs intersect at one end point and $s \subset c, t \subset a b$, and $u \subset \partial \Sigma$. By taking the inner most disc if necessary, we may assume that int $(D) \cap(c \cup a b)=\emptyset$. Let $N(a b)=N(a) \cup N(b)$, $N(a \cap b)=N(a) \cap N(b)$, and $R_{0}, R_{1}, \ldots, R_{n}$ be the set of components of $\Sigma-(c \cup N(a) \cup N(b))$ which are contained in $D$. We set $R_{0}$ to be the region so that $R_{0} \cap c \neq \emptyset$. Then $R_{0} \cap u \neq \emptyset$ if $u \neq \emptyset$. Furthermore, $R_{i} \cap(c \cup \partial \Sigma)=\emptyset$ for $i \geq 1$. By the assumption that $\operatorname{int}(D) \cap(c \cup a b)=\emptyset$, each region $R_{i}$ is a disc. Use the same argument as in (a), each region $R_{i}(i \geq 1)$ has at least four sides and adjacent vertices in $\partial R_{i}(i \geq 0)$ satisfy the alternating principle. Form the same type of graph $G$ in $D$ based on the combinatorics of the regions $R_{i}$ as in (a). Since each region $R_{i}$ is contractible, and $D$ is also contractible, the graph $G$ is a tree. Thus $G$ is either a point or contains two vertices of valency one. Since each $R_{i}(i \geq 1)$ corresponds to a vertex 
of valency at least 2 in the graph $G$, the graph $G$ must be one point which corresponds to the region $R_{0}$. Since $R_{0}$ has valency 1 , by the definition of valency, this shows that the region $R_{0}$ must be a triangle or a quadrilateral in $\Sigma-(a \cup b \cup c)$. By exam the resolution, we see that $R_{0}$ must be one of the three types listed in figure A1 (a1), (a2), and (a3) (the last type (a4) does not occur for the intersection $|(a b) \cap c|$ but it occurs for $|a \cap(b c)|$.) This contradicts the none-existence of positive triangle or positive quadrilateral in the ordered set $(a, b, c)$. QED

Now we come to the proof of theorem 2.4.

Theorem. The multiplication $C S(\Sigma) \times C S(\Sigma) \rightarrow C S(\Sigma)$ sends $C S_{0}(\Sigma) \times$ $C S_{0}(\Sigma) \rightarrow C S_{0}(\Sigma)$ and has the following properties.

(1) It is preserved under the action of the orientation preserving homeomorphisms of the surface.

(2) (commutativity) If $I(\alpha, \beta)=0$, then $\alpha \beta=\beta \alpha$. If $\alpha \beta=\beta \alpha$ and $\alpha \in C S_{0}(\Sigma)$, then $I(\alpha, \beta)=0$.

(3) (associativity) Suppose $\alpha, \beta$, and $\gamma$ are in $C S(\Sigma)$ so that the ordered set $(\alpha, \beta, \gamma)$ contains no positive triangles and positive quadrilaterals, then $\alpha(\beta \gamma)=(\alpha \beta) \gamma$.

(4) (inverse) If $\alpha$ is in $C S_{0}(\Sigma)$ and each component of $\alpha$ intersects $\beta$, then $\alpha(\beta \alpha)=(\alpha \beta) \alpha=\beta$. Furthermore, $I(\alpha, \beta)=I(\alpha, \alpha \beta)=I(\alpha, \beta \alpha)$.

(5) For any integer $k \in \mathbf{Z}_{>0},(\alpha \beta)^{k}=\alpha^{k} \beta^{k}$.

(6)(triangle inequality) $I(\alpha, \beta \gamma) \leq I(\alpha, \beta)+I(\alpha, \gamma)$. The inequality becomes an equality if $(\alpha, \beta, \gamma)$ contains no positive triangles or quadrilaterals. Furthermore, if $\beta \in C S_{0}(\Sigma)$, then $|I(\alpha, \beta \gamma)-I(\alpha, \gamma)| \leq I(\alpha, \beta)$ and $|I(\alpha, \gamma \beta)-I(\alpha, \gamma)| \leq I(\alpha, \beta)$.

Proof. Properties (1) and (5) follow from the definition. See figure A2(b). Property (3) follows from the second part of the previous lemma. Indeed, take $a, b, c$ to be the representatives of $\alpha, \beta$ and $\gamma$ so that the condition in previous lemma (b) holds. Then, by the lemma, both multiplications $\alpha(\beta \gamma)$ and $(\alpha \beta) \gamma$ are obtained from $a \cup b \cup c$ by resolving all intersection points from $a$ to $b$ and from $b$ to $c$.

To see part (4), take $a \in \alpha$ and let $a^{\prime}$ be a parallel copy of $a$ so that $a \cap a^{\prime}=\emptyset$. Take $b \in \beta$ so that it has minimal intersection with both $a$ and $a^{\prime}$. Then we note that there are no positive triangles or positive quadrilaterals in the ordered set $\left(a, b, a^{\prime}\right)$. Thus by the part (b) of the previous lemma, we have $\left[(a b) a^{\prime}\right]=\left[a\left(b a^{\prime}\right)\right]$ and $I\left(a, b a^{\prime}\right)=\left|a \cap\left(b a^{\prime}\right)\right|=|a \cap b|=I(a, b)$. Also we have $I(a, b)=|a \cap b|=\left|a \cap a^{\prime} b\right|=I(a, a b)$.

To see the equation $[a(b a)]=[b]$ when each component of $a$ intersects $b$, 
we use the part (b) of the previous lemma again. By the lemma $a\left(b a^{\prime}\right)$ is obtained from $a \cup b \cup a^{\prime}$ by resolving all intersection points in $a \cap b$ from $a$ to $b$ and all intersection points $a^{\prime} \cap b$ from $a^{\prime}$ to $b$. Thus these two resolutions canceled all the twists made on $b$ (see figure A2). Thus the result follows.

The first part of (2) follows from the definition. To see the second part, suppose otherwise that $\alpha \in C S_{0}$ so that $\alpha \beta=\beta \alpha$ but $I(\alpha, \beta)>0$. We decompose $\alpha$ as a disjoint union $\alpha_{1} \alpha_{2}$ where $I\left(\alpha_{1}, \beta\right)=0$ and each component of $\alpha_{2}$ intersects $\beta$. Now since $\alpha_{1}$ is disjoint from both $\alpha_{2}$ and $\beta$, we have $\beta\left(\alpha_{1} \alpha_{2}\right)=\alpha_{1}\left(\beta \alpha_{2}\right)$ and $\alpha \beta=\alpha_{1}\left(\alpha_{2} \beta\right)$. Thus, by $\alpha \beta=\beta \alpha$, we obtain $\alpha_{2} \beta=\beta \alpha_{2}$. Since each component of $\alpha_{2}$ intersects $\beta$, by property (4), $\beta=\alpha_{2}\left(\beta \alpha_{2}\right)=\alpha_{2}\left(\alpha_{2} \beta\right)=\left(\alpha_{2}\right)^{2} \beta$ where the last equality follows from property (3). Now by property (4) again, $I(\beta, \beta)=I\left(\beta,\left(\alpha_{2}^{2}\right) \beta\right)=I\left(\beta, \alpha_{2}^{2}\right)$ $=2 I\left(\beta, \alpha_{2}\right) \neq 0$. This is a contradiction.

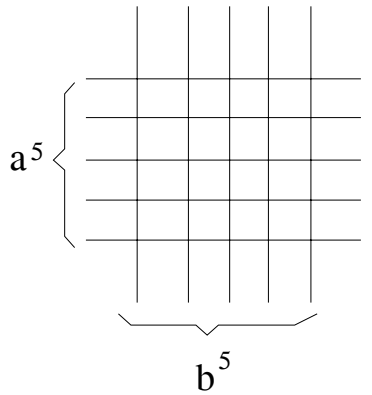

part (a)

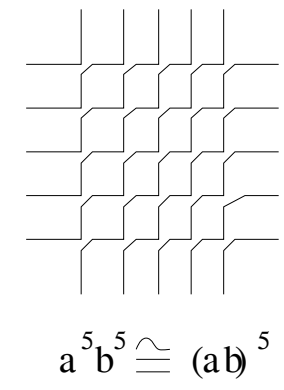

b

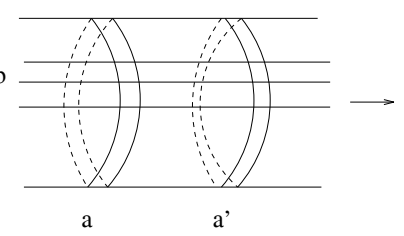

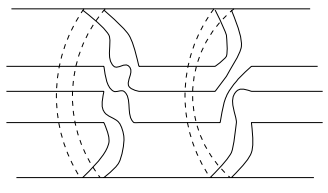

a(ba')

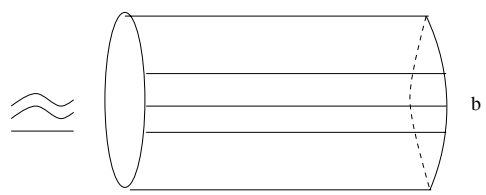

b

$\operatorname{part}(\mathrm{b})$

Figure A2

To see property (6), the triangle inequality $I(\alpha, \beta \gamma) \leq I(\alpha, \beta)+I(\alpha, \gamma)$ holds by the definition of the resolution. Furthermore, part (b) of the previous lemma says that the inequality $I(\alpha, \beta \gamma) \leq I(\alpha, \beta)+I(\alpha, \gamma)$ becomes an equality if $(\alpha, \beta, \gamma)$ contains no positive triangles and positive quadri- 
laterals. To see the last part of (6), we need to show that $I(\alpha, \gamma) \leq$ $I(\alpha, \beta)+I(\alpha, \beta \gamma)$ and $I(\alpha, \gamma) \leq I(\alpha, \beta)+I(\alpha, \gamma \beta)$. For simplicity, let us prove the first inequality that $I(\alpha, \gamma) \leq I(\alpha, \beta)+I(\alpha, \beta \gamma)$. A similar argument proves the second inequality. Note that by the triangle inequality $I(\alpha, \beta)+I(\alpha, \beta \gamma) \geq I(\alpha,(\beta \gamma) \beta)$. Now write $\beta=\beta_{1} \beta_{2}$ where $\beta_{1}$ consists of components of $\beta$ disjoint from $\gamma$ and $\beta_{2}$ consists of components of $\beta$ which intersect $\gamma$. Thus by property $(4),(\beta \gamma) \beta=\left(\beta_{1}\right)^{2} \gamma$ where $I\left(\beta_{1}, \gamma\right)=0$. Thus $I(\alpha,(\beta \gamma) \beta)=I\left(\alpha,\left(\beta_{1}\right)^{2}\right)+I(\alpha, \gamma) \geq I(\alpha, \gamma)$. This ends the proof.

\section{References.}

[Bo] Bonahon, F.: Bouts des variétés hyperboliques de dimension 3. Ann. of Math. (2) 124 (1986), no. 1, 71-158.

[Bo1] Bonahon, F.: Earthquakes on Riemann surfaces and on measured geodesic laminations. Trans. Amer. Math. Soc. 330 (1992), no. 1, 6995.

[De] Dehn, M.: Papers on group theory and topology. J. Stillwell (eds.). Springer-Verlag, Berlin-New York, 1987.

[FLP] Fathi, A., Laudenbach, F., Poenaru, V.: Travaux de Thurston sur les surfaces. Astérisque 66-67, Société Mathématique de France, 1979.

[HM] Hubbard, J. and Masur, H.: Quadratic differentials and foliations. Acta Math. 142 (1979), no. 3-4, 221-274.

[Lu] Luo, F., Simple loops on surfaces and their intersection numbers, Journal of Diff. Geom., to appear.

[Pa] Papadopoulos, A.: On Thurston's boundary of Teichmller space and the extension of earthquakes. Topology Appl. 41 (1991), no. 3, 147177 .

[PH] Penner, R. and Harer J.: Combinatorics of train tracks. Annals of Mathematics Studies, 125. Princeton University Press, Princeton, NJ, 1992.

[Re] Rees, M., An alternative approach to the ergodic theory of measured foliations on surfaces, Ergodic Theory and Dynamic Systems, 1 (1981), no. 4, 461-488. 
[Th1] Thurston, W., On the geometry and dynamics of diffeomorphisms of surfaces, Bul. Amer. Math. Soc. 19 (1988) 417-438.

[Th2] Thurston, W., Geometry and topology of 3-manifolds, Princeton University lecture notes, 1976.

FENG LUO

Department of Mathematics

RUTGERS UNIVERSITY

fluo@math.rutgers . edu

Richard Stong

Department of Mathematics

Rice UNIVERsity

stong@math.rice.edu 
F. Luo and R. Stong 